ARTICLE

\title{
NUDT2 initiates viral RNA degradation by removal of 5'-phosphates
}

\author{
Beatrice T. Laudenbach 1,2,14, Karsten Krey (10 1,14, Quirin Emslander ${ }^{1,14}$, Line Lykke Andersen,
} Alexander Reim (1) ${ }^{3}$, Pietro Scaturro (1) 1,4, Sarah Mundigl ${ }^{1}$, Christopher Dächert ${ }^{5,6}$, Katrin Manske ${ }^{7}$, Markus Moser ${ }^{8,9}$, Janos Ludwig ${ }^{10}$, Dirk Wohlleber (10) ${ }^{7}$, Andrea Kröger ${ }^{11,12}$, Marco Binder (iD ${ }^{5}$ \& Andreas Pichlmair (1) 1,2,13凶

While viral replication processes are largely understood, comparably little is known on cellular mechanisms degrading viral RNA. Some viral RNAs bear a 5'-triphosphate (PPP-) group that impairs degradation by the canonical $5^{\prime}-3^{\prime}$ degradation pathway. Here we show that the Nudix hydrolase 2 (NUDT2) trims viral PPP-RNA into monophosphorylated (P)-RNA, which serves as a substrate for the $5^{\prime}-3^{\prime}$ exonuclease XRN1. NUDT2 removes $5^{\prime}$-phosphates from PPP-RNA in an RNA sequence- and overhang-independent manner and its ablation in cells increases growth of PPP-RNA viruses, suggesting an involvement in antiviral immunity. NUDT2 is highly homologous to bacterial RNA pyrophosphatase $\mathrm{H}(\mathrm{RppH})$, a protein involved in the metabolism of bacterial mRNA, which is $5^{\prime}$-tri- or diphosphorylated. Our results show a conserved function between bacterial $\mathrm{RppH}$ and mammalian NUDT2, indicating that the function may have adapted from a protein responsible for RNA turnover in bacteria into a protein involved in the immune defense in mammals.

\footnotetext{
${ }^{1}$ Technical University of Munich, School of Medicine, Institute of Virology, 81675 Munich, Germany. ${ }^{2}$ Innate Immunity Laboratory, Max-Planck Institute of Biochemistry, Martinsried/Munich, Germany. ${ }^{3}$ Department of Proteomics and Signal transduction, Max-Planck Institute of Biochemistry, Martinsried/ Munich, Germany. ${ }^{4}$ Leibniz Institute for Experimental Virology (HPI), Hamburg, Germany. ${ }^{5}$ Research Group "Dynamics of Early Viral Infection and the Innate Antiviral Response" (division F170), German Cancer Research Center, Heidelberg (DKFZ), Heidelberg, Germany. ${ }^{6}$ Faculty of Biosciences, Heidelberg University, 69120 Heidelberg, Germany. ${ }^{7}$ Technical University of Munich, School of Medicine, Institute of Molecular Immunology, Munich, Germany.

${ }^{8}$ Department of Molecular Medicine, Max-Planck Institute of Biochemistry, Martinsried/Munich, Germany. ${ }^{9}$ Technical University of Munich, School of Medicine, Institute of Experimental Hematology, Munich, Germany. ${ }^{10}$ Institute of Clinical Chemistry and Clinical Pharmacology, University Hospital Bonn, Bonn, Germany. ${ }^{11}$ Otto von Guericke University Magdeburg, Institute for Medical Microbiology, Magdeburg, Germany. ${ }^{12}$ Helmholtz Centre for Infection Research, Innate Immunity and Infection, Braunschweig, Germany. ${ }^{13}$ German Center for Infection Research (DZIF), Munich partner site, Munich, Germany.

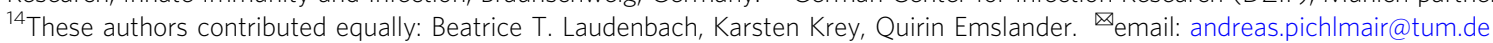


A Il living organisms use RNA as the messenger molecule to translate their DNA encoded genetic information into proteins. Besides trans-activating sequences encoded on messenger (m)RNA and the subcellular localization of the RNA molecule, the abundance of mRNA is the primary determinant to modulate gene expression. mRNA abundance is regulated based on transcription and the stability of the molecule. In eukaryotes, the most prominent and evolutionary conserved processes that modulate the stability of mRNA are $5^{\prime}$-to- $3^{\prime}$ RNA degradation by the cellular exonuclease 1 (XRN1) and $3^{\prime}$-to- $5^{\prime}$ degradation by the RNA exosome complex. Eukaryotic mRNAs are protected from $5^{\prime}$-to-3' degradation by a $5^{\prime}$-m7G cap structure, cotranscriptionally added to the mRNA molecule. To proceed with the $5^{\prime}$-to- $3^{\prime}$ degradation process, the mRNA cap needs to be removed by the decapping protein 2 (DCP2) to generate a monophosphorylated (P-)RNA substrate, which can then be degraded by XRN1 (Fig. 1a) ${ }^{1,2}$. Genomic RNAs and RNA transcripts of many viruses, including Orthomyxo-, Arena-, Paramyxo- and Bunyaviruses, do not bear a $5^{\prime}$-cap but present a $5^{\prime}$ - triphosphate (PPP-) group. This terminal PPP- moiety linked to double-stranded RNA serves as a pathogen-associated molecular pattern (PAMP) sensed by the cellular helicase RIG-I, which activates a MAVS-dependent innate immune signaling cascade. Eventually, this culminates in the accumulation of type-I interferon (IFN) and IFN-stimulated genes and is critical to prevent virus spread $^{3}$. It is currently unclear why some viruses bear terminal triphosphates despite the selective pressure of the innate immune system. However, a possible explanation may be the increased stability of PPP-RNA that inhibits $5^{\prime}$-to- $3^{\prime}$ degradation by XRN1. Interestingly, despite the stabilizing PPP-group on viral RNA, specific highly secondary structured viral RNA sequences expressed by Flavi-, Bunya-, and Arenaviruses impair the activity of XRN1 $1^{4,5}$. Moreover, the depletion of XRN1 increases the stability of viral RNA ${ }^{6}$. Collectively, this suggests that $5^{\prime}$-to- $3^{\prime}$ RNA degradation processes are operative in the case of viral RNA. In analogy to the eukaryotic mRNA degradation system, however, the degradation of viral PPP-RNA would require a processing step that removes the PPP-group. Indeed, recently the a

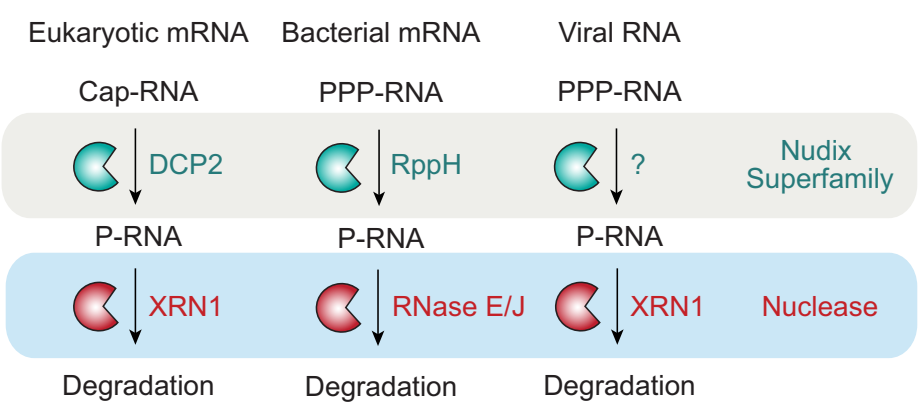

C

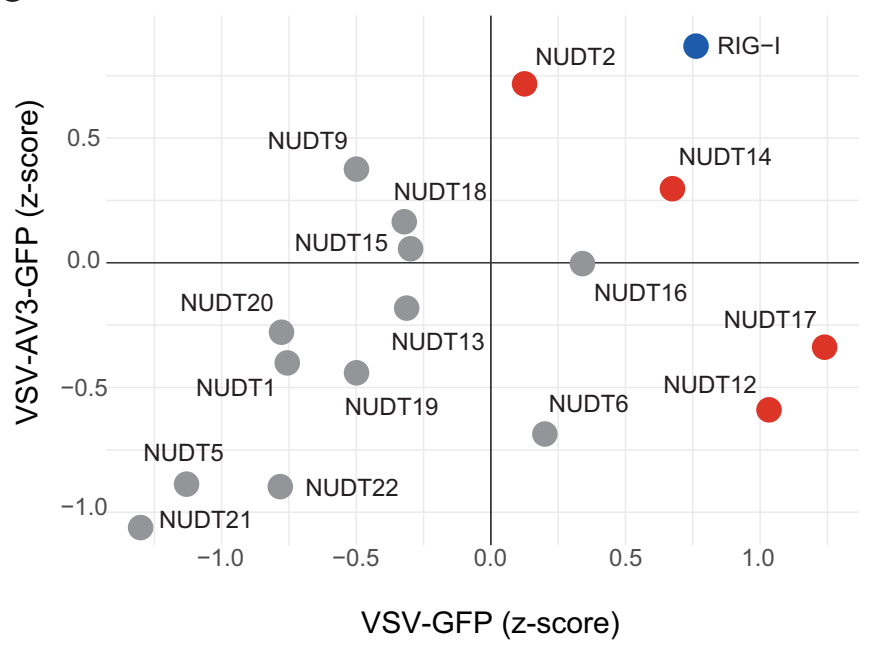

b

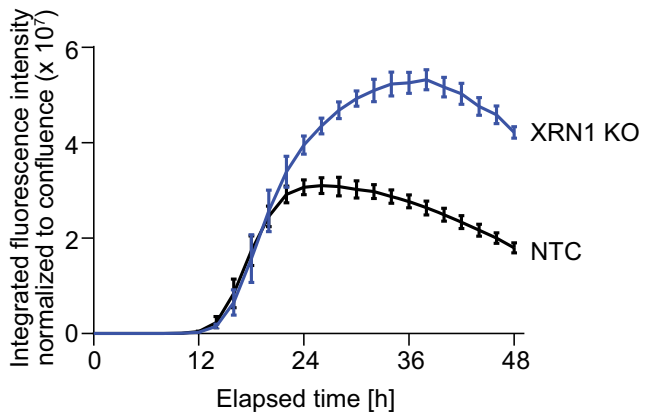

d

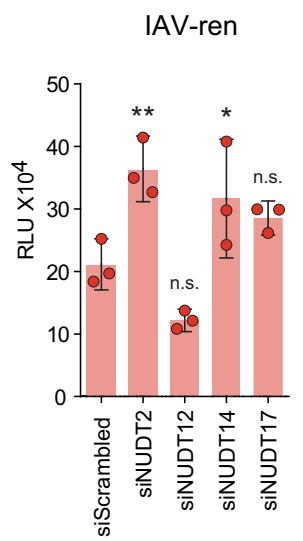

Fig. 1 Influence of Nudix depletion on virus growth. a Schematic representation of processes involved in 5'-to-3' RNA degradation. Eukaryotic and bacterial triphosphorylated messenger (m)RNA are processed by proteins DCP2 and RppH, respectively, to generate monophosphorylated substrates for the 5'-3' exonuclease XRN1, RNase E or RNase J. We hypothesize that a mammalian Nudix hydrolase could prepare viral RNA for degradation by removing pyrophosphate groups. b Hep3B cells with a CRISPR/Cas9-mediated KO of XRN1 or Hep3B cells transduced with a non-targeting-control vector (NTC) were infected with VSV-GFP at MOI 0.01 for $48 \mathrm{~h}$. GFP expression was continuously quantified by live-cell microscopy. The data points are displayed as the mean of five technical replicates \pm SD. One representative experiment of three is shown. $\mathbf{c}$ HeLa cells transfected with siRNA pools targeting the indicated Nudix gene for $48 \mathrm{~h}$ were infected with VSV-GFP ( $x$-axis) or VSV-AV3-GFP ( $y$-axis). Twenty-four hours after infection, GFP expression was analyzed by fluorometric analysis. The displayed $z$-scores were calculated from three independent reactions. $\mathbf{d}$ HeLa cells were treated with siRNA pools for $48 \mathrm{~h}$ targeting the transcript of NUDT2, NUDT12, NUDT14, NUDT17, or non-targeting siRNA control (siScrambled) and infected with Influenza A reporter virus expressing the renilla luciferase (IAV-ren) at $\mathrm{MOI}$ 1. Forty-eight hours post-infection, renilla luciferase activity was analyzed. The means of three biological replicates $\pm \mathrm{SD}$ are shown; ${ }^{\star \star} p=0.004,{ }^{\star} p=0.03$ as analyzed by two-way analysis of variance (ANOVA) statistics. RLU relative light units. n.s. not significant. 
triphosphatases RNA/RNP complex-1-interacting phosphatase (DUSP11) and Decapping and exoribonuclease protein (DXO, also known as DOM3Z) were shown to dephosphorylate PPPRNA to P-RNA that can be further degraded by $\mathrm{XRN1}^{7-9}$. However, the mechanism of PPP-RNA dephosphorylation and the substrate specificity of DUSP11 is not yet well understood.

Similar to viral RNA, prokaryotic mRNA also bears a $5^{\prime}$-PPP or $5^{\prime}$-PP group ${ }^{10,11}$. In bacteria, the $5^{\prime}$ phosphates can be removed by the RNA pyrophosphohydrolase (RppH) to prepare RNA for degradation by the bacterial $5^{\prime}$-to- $3^{\prime}$ endoribonuclease RNase $\mathrm{E}$ or $5^{\prime}$ exonucleases (e.g., RNase J11-13 (Fig. 1a)). Interestingly, both mammalian DCP2 and bacterial RppH belong to the superfamily of Nudix hydrolases ${ }^{14}$, a protein family that is highly diverse in terms of sequence, domain organization, and substrate specificity ${ }^{15}$. Nudix proteins are characterized by a Nudix hydrolase domain, which contains characteristic catalytic and metal-binding amino acids ${ }^{16}$. They mostly function as pyrophosphohydrolases active towards substrates with the structure NDP$\mathrm{X}$ (nucleoside diphosphate linked to another moiety, $X$ ), usually resulting in NMP (nucleoside monophosphate) and P-X as products ${ }^{16-18}$. Since Nudix hydrolases process mammalian and bacterial mRNAs, we tested whether mammalian Nudix hydrolases may have dephosphorylation activity on PPP-RNA to initiate further exonucleolytic processing. Understanding such viral RNA degradation mechanisms is essential to further study cellular antiviral principles that are in place to limit the spread and pathogenicity of infectious pathogens and their involvement in antiviral immunity.

\section{Results}

Nudix hydrolases have an impact on virus growth. To establish a screening system that allows testing for $5^{\prime}$-to- $3^{\prime}$ dependent RNA degradation in virus-infected cells, we first evaluated whether CRISPR/Cas9-mediated depletion of XRN1 affects the replication of PPP-RNA-generating vesicular stomatitis virus expressing green fluorescent protein (VSV-GFP). Automated live-cell microscopy of VSV-GFP infected cells showed a distinct increase of GFP signal in XRN1 targeted cells as compared to controls (Fig. 1b), indicating that VSV-GFP is restricted by a $5^{\prime}$ to-3' exonuclease dependent pathway. We hypothesized that any Nudix hydrolase that may be involved in PPP-RNA processing should similarly negatively regulate virus growth. We, therefore, used siRNAs to deplete mammalian Nudix proteins, which are known to play a role in nucleotide metabolism ${ }^{17}$ (Supplementary Table 1) and quantified the viral growth of VSV-GFP and the IFN-stimulating variant VSV-AV3-GFP ${ }^{19}$. Compared to the control treatment, depletion of the PPP-RNA-sensing pattern recognition receptor RIG-I led to increased GFP expression after infection with either of the two viruses, confirming the validity of this approach (Fig. 1c). Notably, the depletion of four Nudix hydrolases (NUDT2, NUDT12, NUDT14, and NUDT17) led to an increase in VSV-GFP or VSV-AV3-GFP growth. As tested by cell titer glow assay, cell viability was not affected by the depletion of these Nudix proteins (Supplementary Fig. 1a). Depletion of NUDT5, NUDT19, NUDT21, and NUDT22 showed a negative impact on VSV growth (Fig. 1c). For NUDT19 and NUDT22, this phenotype could potentially be explained by reduced cell viability after depletion of these Nudix hydrolases. In total, we selected four Nudix hydrolases (NUDT2, -12, -14, and -17), which showed the highest suppressive effect on either of the two viruses for further experiments. We confirmed the knockdown efficiency of NUDT2, -12, -14, and -17 siRNA treatment in HeLa cells by RTqPCR and western blotting (NUDT2) (Supplementary Fig. 1b, c). We used renilla-luciferase-expressing Influenza A virus (IAVren) as an alternative PPP-RNA generating virus to confirm the antiviral activity of the four NUDTs (Fig. 1d). While the depletion of NUDT12 and NUDT17 did not significantly increase the renilla signal in this cell type, the depletion of NUDT2 and -14 led to a significant increase (Fig. 1d).

NUDT2 releases phosphates from a triphosphorylated RNA substrate. To characterize the four selected Nudix hydrolases for their potential ability to dephosphorylate PPP-RNA, we generated recombinant NUDT2, -12, -14, and -17 in E. coli. As controls, we mutated the metal-coordinating glutamic acid residue (E) in their conserved Nudix hydrolase domain motif (NUDT2 E58A, NUDT12 E369A, NUDT14 E121A, and NUDT17 E124A20. Successful purification of the recombinant proteins was confirmed by SDS-PAGE and mass spectrometry (Supplementary Fig. 2a, b). As expected, co-incubation with in vitro transcribed RNAs showed that the purified proteins and recombinant calf intestinal phosphatase (CIP) did not have RNase activity (Fig. 2a). RNase A-treated RNA, however, was clearly degraded. We next tested whether the recombinant Nudix proteins can dephosphorylate PPP-RNA. For this, we used thin-layer chromatography (TLC), which allows visualization of released $\gamma$ phosphates from $\gamma^{-32} \mathrm{P}$-labeled single-stranded 44-mer PPPRNA substrate. Notably, NUDT2 released substantial amounts of $\gamma$-phosphate (Fig. $2 \mathrm{~b}, \gamma^{-32} \mathrm{P}$ ) and led to almost complete dephosphorylation of the substrate RNA (Fig. 2b, RNA). In contrast, the other tested Nudix proteins (NUDT12, NUDT14, and NUDT17) only showed a minute release of $\gamma^{-32} \mathrm{P}$. The NUDT2-dependent dephosphorylation was time-dependent (Fig. 2c) and required Nudix hydrolase activity since the NUDT2 E58A mutant did not release radioactively labeled phosphates or change RNA substrate labeling (Fig. 2d). Dephosphorylation was also apparent when using double-stranded RNA substrate, which was generated by annealing antisense RNA to the radioactively labeled 44-mer substrate (dsRNA) and by using radioactively labeled IVT4 (hairpin RNA) as substrate (Fig. 2e). Bacterial RppH, known for its activity to release $\gamma$-phosphates from unpaired RNA $5^{\prime}$ overhangs ${ }^{11}$, showed a similar, although less pronounced release of phosphates from single-stranded PPP-RNA substrates (Fig. 2e). These experiments indicated that human NUDT2, like bacterial $\mathrm{RppH}$, has dephosphorylation activity, pointing towards NUDT2 as a candidate protein involved in PPP-RNA degradation and antiviral immunity.

Interestingly, sequence alignment analysis suggests that NUDT2 is highly conserved in evolutionary distant eukaryotic species (Supplementary Fig. 2c). Notably, after sequence alignment of all human Nudix hydrolases, NUDT2 shows the highest sequence similarity to bacterial $\mathrm{RppH}$, further pointing towards a conserved function of both proteins (Fig. 2f). The crystal structures of RppH and NUDT2 have been solved ${ }^{20,21}$, and alignment of these structures revealed striking similarities in which the active site of NUDT2 resembles the RNA binding pocket of $\mathrm{RppH}$ (Fig. 2g). It exhibits a negatively charged interface required for positioning the positively charged magnesium ions needed to coordinate the RNA's PPP-group. Furthermore, the RNA binding pocket contains a large, positively charged channel bearing space for the negatively charged RNA phosphate backbone (Fig. 2h).

NUDT2 is active on a broad range of PPP-RNA substrates. We focused on NUDT2 as a candidate protein involved in viral RNA degradation and therefore assessed the ability of NUDT2 to release phosphates from different substrates. To this aim, we used a time-resolved enzymatic assay to compare phosphate release from PPP-RNA. Titration of NUDT2 suggested an optimal signal-to-noise ratio when $600 \mathrm{nM}$ of NUDT2 were used 
a

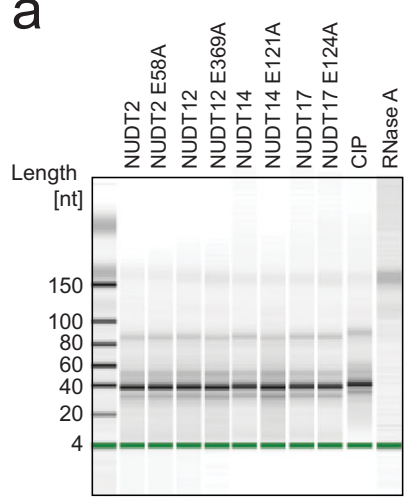

e

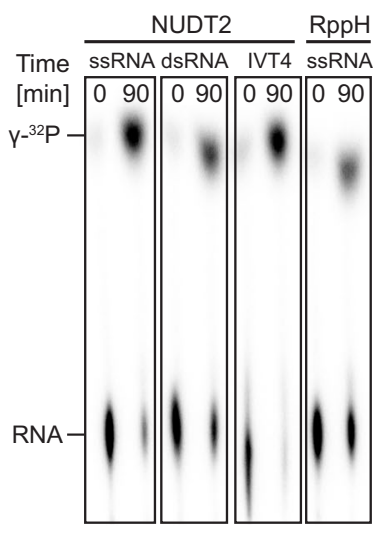

h

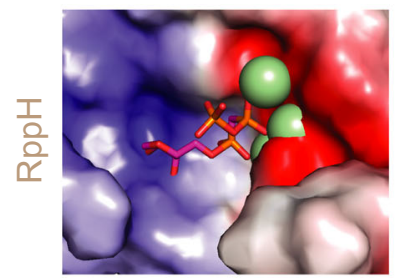

b

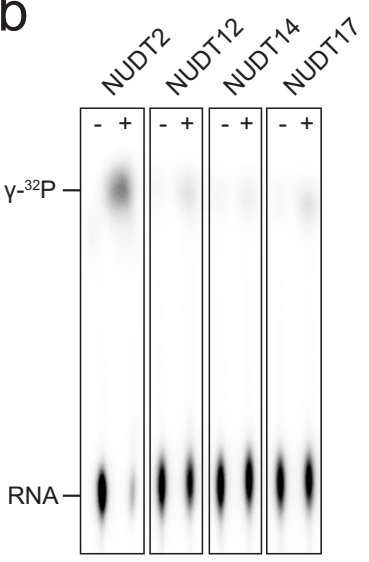

f
C

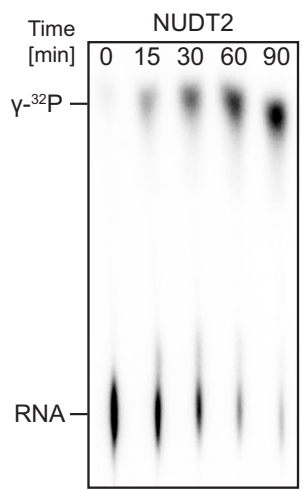

d

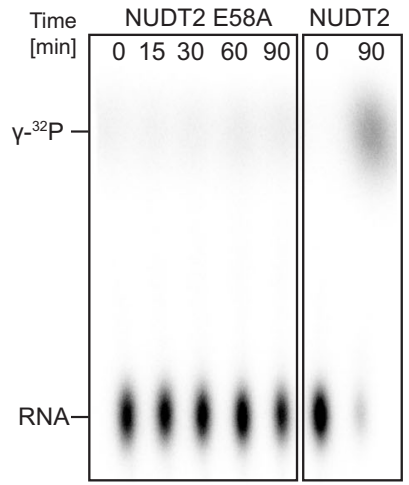

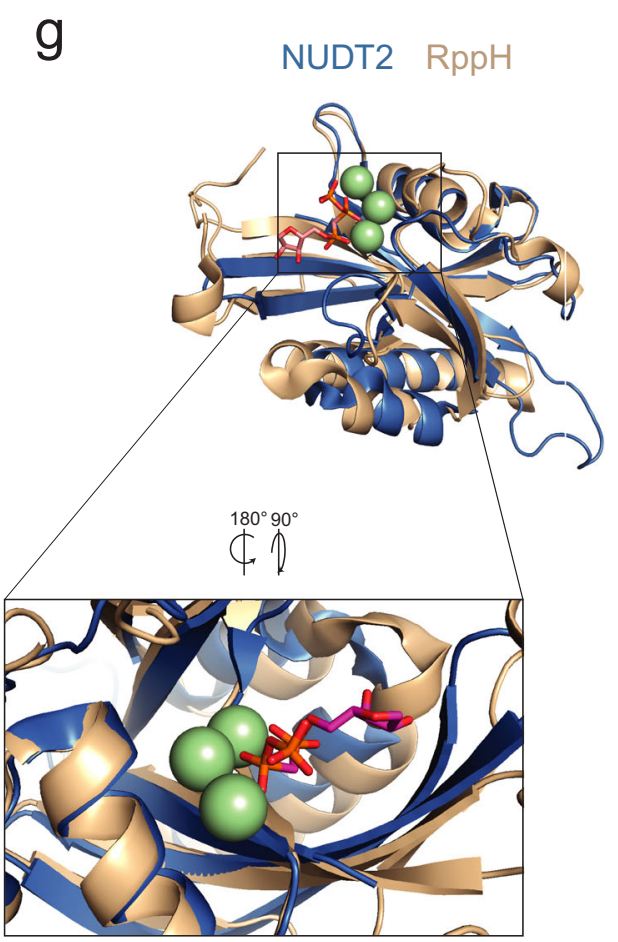

NUDT2 RppH

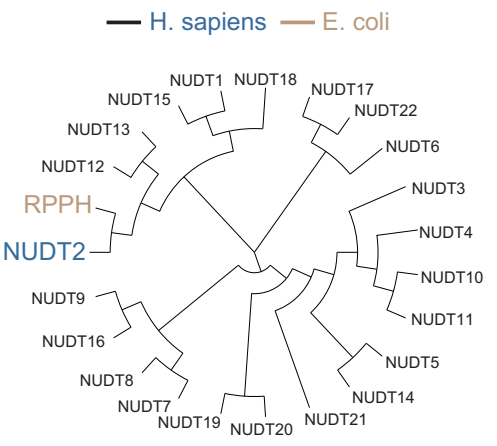

Fig. 2 Identification of NUDT2 as PPP-RNA phosphatase. a RNA was incubated for $3 \mathrm{~h}$ with the indicated Nudix hydrolase, and the integrity of the RNA was determined using a Bioanalyzer. One representative experiment of three is shown. $\mathbf{b} \gamma$-32P-radiolabeled in vitro transcribed 44-mer ssRNA substrate was left untreated or incubated for 90 min with 600 nM NUDT2, NUDT12, NUDT14 or NUDT17 (+) or was left untreated ( - ). Reaction mixtures were analyzed by TLC and autoradiography. $\gamma$-32p: radiolabeled $\gamma$-phosphate; RNA: remaining input RNA after incubation. c As (b) but incubation for the time indicated at the top of the panel. $600 \mathrm{nM}$ NUDT2 were used. d As (c) but $600 \mathrm{nM}$ NUDT2 E58A or NUDT2 were used. e As (b) but $\gamma$-32P-radiolabeled in vitro transcribed single-stranded (ss-) or double-stranded (ds-) RNA generated by annealing an antisense oligo, or a RIG-I activating hairpin RNA (IVT4) were incubated with $600 \mathrm{nM}$ NUDT2 and $5 \mathrm{U} \mathrm{RppH}$, respectively. f Phylogenetic analysis of all human Nudix hydrolases and bacterial RppH was performed using MAFFT. $\mathbf{g}$ Structural alignment of the crystal structures of RppH (brown) with an RNA substrate (red sticks) (PDB: 4S2Y) and NUDT2 (blue) (PDB: 3U53) using PYMOL ${ }^{57}$. The green-colored magnesium ions spheres are essential for hydrolase activity. $\mathbf{h}$ Visualization of the electrostatic surface charges of RppH and NUDT2 displayed with the aligned RNA of the RppH structure using PYMOL. Acidic amino acids are depicted in red, and basic residues are shown in blue.

(Supplementary Fig. 3a). Phosphatase activity of CIP was higher, probably due to more efficient or more complete dephosphorylation of the RNA substrate. As expected, NUDT2 mediated phosphate release is dependent on a glutamic acid at the catalytic site since the NUDT2 E58A mutant or control proteins (NUDT14, NUDT14 E121A) failed to yield a considerable signal (Fig. 3a).

To investigate the reactivity of NUDT2 on different substrates, we designed RNAs with or without different $5^{\prime}$ single-stranded overhangs and variable nucleotides at the first $(A, G)$ and the second position (A, G, U, and C) of the RNA (Fig. 3b). NUDT2 processed all constructs, irrespective of the length of the $5^{\prime}$ singlestranded overhang (Fig. 3c). Moreover, NUDT2 was also able to dephosphorylate RNA substrates with adenines at the $5^{\prime}$ end and $5^{\prime}$ single-stranded RNA overhang starting with adenines (Fig. $3 \mathrm{c}$ ). As expected, the control NUDT2 E58A did not mediate significant phosphate release (Supplementary Fig. 3b). NUDT2 did not degrade the RNA substrates (Fig. 3d), confirming the lack of nuclease activity of NUDT2. Importantly, NUDT2 did not release any phosphates from single nucleotides (rATP, rGTP, 


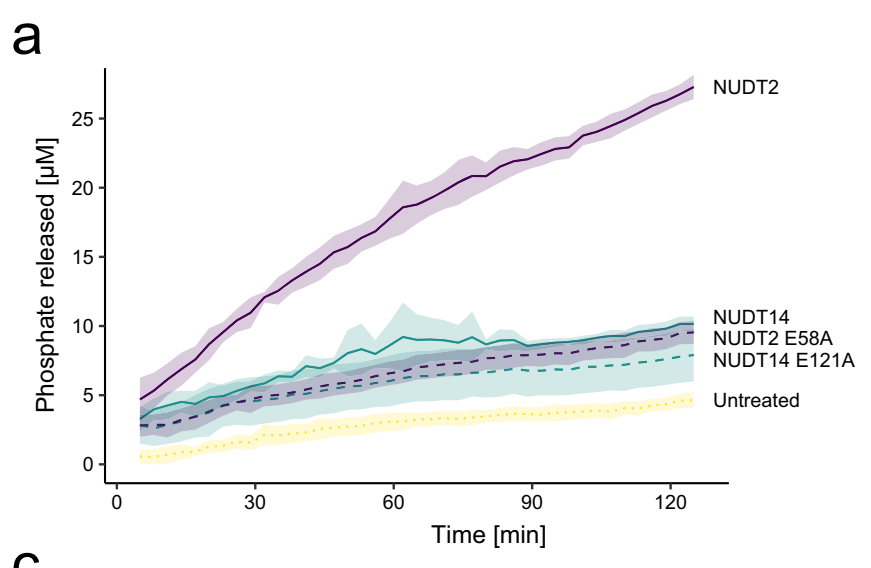

C

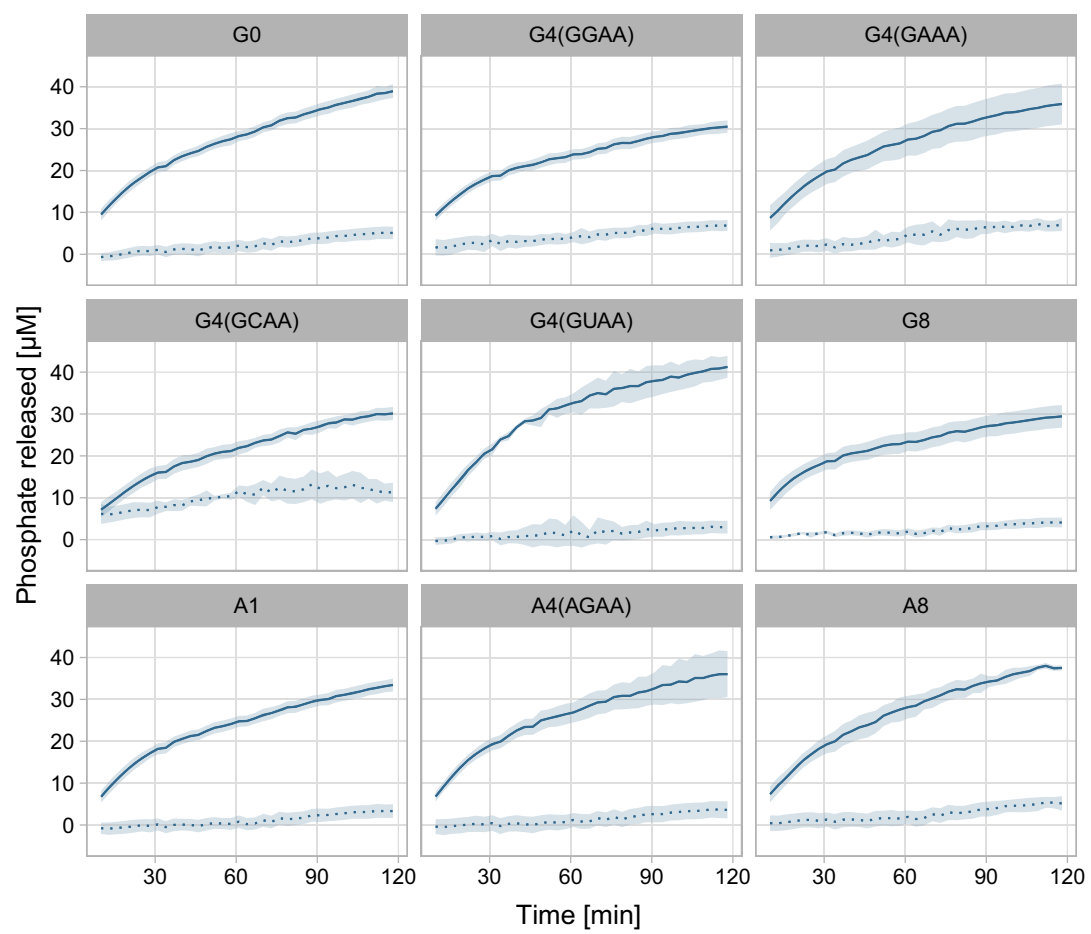

b<smiles>C=CC1CO1</smiles><smiles>[Mg][Mg]</smiles><smiles>CC(=O)O</smiles>

G4 (GGAA)

G4 (GAAA)<smiles>CC(=O)O</smiles><smiles>CC(=O)O</smiles>

G4 (GUAA)

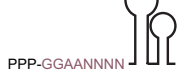

G8<smiles></smiles><smiles>C[13CH]=O</smiles>
A4 (AGAA)<smiles>[Mg][Mg]</smiles>

d

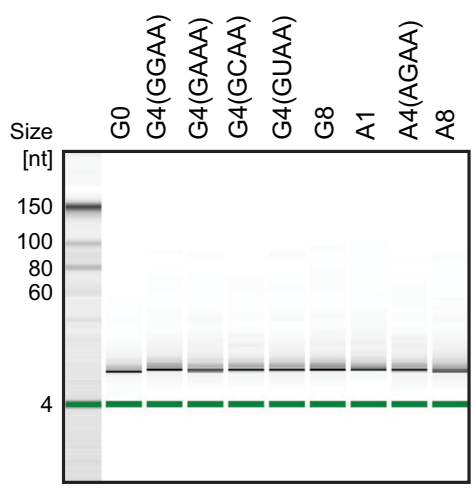

e

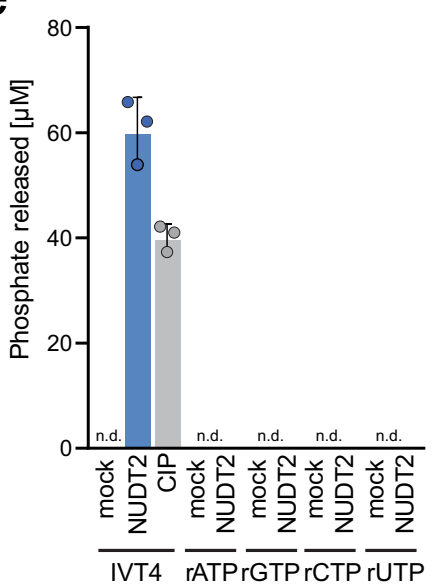

Fig. 3 NUDT2 is active on a broad range of substrates. a EnzChek assay to quantify released phosphate from in vitro transcribed double-stranded triphosphorylated hairpin RNA (IVT4) left untreated (untreated) or incubated with recombinant NUDT2, NUDT E58A, NUDT14, or NUDT14 E121A $(600 \mathrm{nM})$, as indicated. Colorimetric measurements of the samples were performed every 3 min. The line graph shows the mean of three independent experiments with the ribbon indicating \pm SD. $\mathbf{b}$ Illustration of in vitro transcribed RNA substrates with varying starting nucleotides with or without paired $5^{\prime}$ overhangs. c RNA substrates depicted in (b) were incubated without (dotted line) or with (solid line) NUDT2 at a concentration of $600 \mathrm{nM}$ over a time course of $2 \mathrm{~h}$, and phosphate release was evaluated using the EnzChek assay. Spectrophotometric measurements to quantify phosphate release were performed every $3 \mathrm{~min}$. The line graph shows the mean of three independent experiments with the ribbon indicating \pm SD. $\mathbf{d}$ The indicated RNA was incubated with NUDT2 (600 nM) for $3 \mathrm{~h}$, and the integrity was analyzed using an Agilent small RNA chip and a Bioanalyzer. e The indicated ribonucleoside triphosphates $(2.5 \mu \mathrm{M})$ and the positive control IVT4 were incubated for $3 \mathrm{~h}$ with NUDT2 $(600 \mathrm{nM})$, and phosphate release was determined by malachite green assay. The mean of three independent reactions \pm SD is shown.

rUTP, and rCTP) at comparable molarity (Fig. 3e), indicating that NUDT2 prefers polynucleotides as substrates. We concluded from these results, that NUDT2 is dephosphorylating PPP-RNAs irrespective of the first nucleotide (A or $\mathrm{G}$ ), the $5^{\prime}$-terminus sequence, and the base-pairing of the $5^{\prime}$-end (single/doublestranded) of the substrate. Regarding substrate preference, NUDT2 appears to be different from $\mathrm{RppH}$, which requires an unpaired $5^{\prime}$ overhang for its activity ${ }^{11}$ (Supplementary Fig. 3c).
NUDT2 releases monophosphates in a sequential manner. To assess the mode of phosphate removal by NUDT2, we synthesized PPP-dinucleotides (PPP-GpA and PPP-ApG) and tested how NUDT2 processes these substrates. Notably, NUDT2 sequentially released single phosphates resulting in a di-phosphorylated dinucleotide intermediate that is further converted into a mono-phosphorylated product (Fig. 4a-c). This contrasts with $\mathrm{RppH}$, which predominantly releases 
a

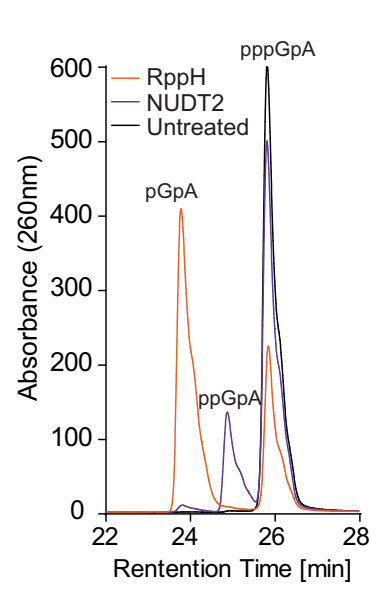

b

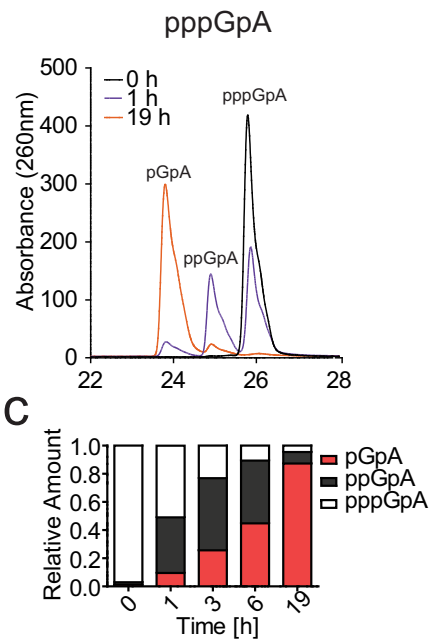

d
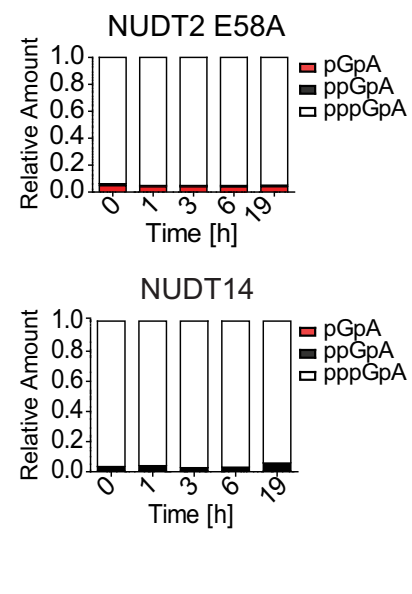

e

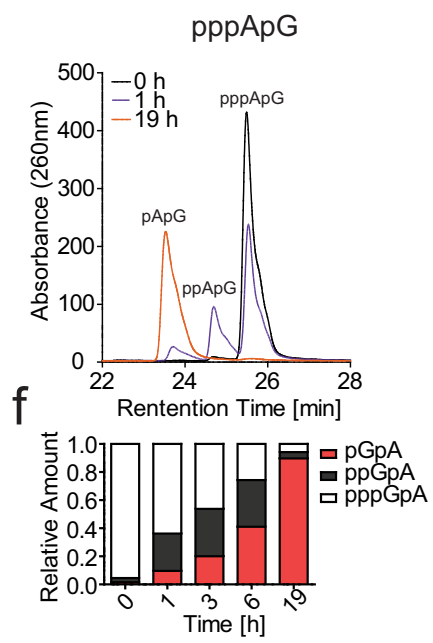

Fig. 4 NUDT2 sequentially releases phosphates from PPP-RNA. a A triphosphorylated RNA dinucleotide (pppGpA) was treated with NUDT2 (600 nM) or $\mathrm{RppH}(5 \mathrm{U})$ for $2 \mathrm{~h}$ at $37^{\circ} \mathrm{C}$. Additionally, the reference pppGpA was incubated in the same reaction buffer (untreated). Products were analyzed by reverse-phase HPLC. The absorbance at $260 \mathrm{~nm}$ at the indicated retention time is shown. $\mathbf{b}$ As (a) incubation with $600 \mathrm{nM}$ NUDT2 for the indicated time. c As (b) but quantification of the generated RNA after incubation of pppGpA with NUDT2 for the indicated time. Quantification was performed by the integration of the corresponding peak area. d As (b) but incubation with NUDT2 E58A or NUDT14. e As (b), but the triphosphorylated dinucleotide pppApG was used as substrate. $\mathbf{f}$ As (e) but quantification of the generated RNA after incubation of pppApA with NUDT2 for the indicated time. Quantification was performed by the integration of the corresponding peak area.

pyrophosphates from PPP-GpA in a one-step reaction without detectable PP-RNA intermediate ${ }^{22}$ (Fig. 4a). Neither NUDT2 E58A mutant nor NUDT14 changed the phosphorylation status of PPP-GpA, confirming the activity of NUDT2 in this assay (Fig. 4d). Removal of monophosphates by NUDT2 was also observed when using a PPP-ApG nucleotide as substrate, further corroborating that RNAs containing guanosines or adenosines at the $5^{\prime}$ end are similarly dephosphorylated by NUDT2 (Figs. 3c, 4e, f).

NUDT2 and XRN1 cooperate for PPP-RNA degradation. We next tested whether NUDT2 can prepare PPP-RNA for degradation by the canonical XRN1 dependent $5^{\prime}$-to-3' RNA degradation machinery. As a substrate, we used Hepatitis $C$ virus (HCV) encoding PPP-RNA. Incubation with NUDT2 or XRN1 did not affect the stability of the PPP-HCV RNA (Fig. 5a, b), indicating that the PPP-group protects RNA from degradation by XRN1 and that NUDT2 does not have exonuclease activity. Notably, co-incubation of HCV PPP-RNA with NUDT2 and XRN1 led to RNA degradation, confirming that the two proteins can act in concert to degrade PPP-RNA (Fig. 5a, b). Control experiments using bacterial $\mathrm{RppH}$, as well as $\mathrm{RppH} / \mathrm{XRN1}$ cotreatment gave similar results. These data demonstrate the functional synergy between NUDT2 and XRN1 in a $5^{\prime}$-to- $3^{\prime}$ degradation pathway of PPP-RNA. As NUDT2 consecutively dephosphorylates PPP-RNA (Fig. 4a, b, e), this suggests that mammalian NUDT2 can, in principle, process PPP-RNA to prepare it for degradation by XRN1.

To extend this in vitro model to physiological conditions, we evaluated the activity of NUDT2 mammalian cells. In human cells, NUDT2 localizes to both the nucleus and the cytoplasm (Supplementary Fig. 4a), which suggests that NUDT2 can be biologically active in both compartments. NUDT2 precipitated from HEK293T cells could release significantly more phosphates from a PPP-RNA compared to NUDT2 E58A mutant or control proteins (Supplementary Fig. 4b, c), indicating that mammalian cell-expressed NUDT2 also has phosphatase activity.
Targeting endogenous NUDT2 in HeLa cells using CRISPR/ Cas9 did not lead to any phenotypic alteration of cells compared to control treatments (Supplementary Fig. 6a). We transfected in vitro transcribed HCV-luciferase PPP-RNA into these cells and analyzed its abundance over time using RT-qPCR. As expected, the abundance of HCV-Luc PPP-RNA declined over time (Fig. 5c). Remarkably, in the absence of NUDT2, HCV-Luc PPP-RNA was considerably more stable, suggesting that NUDT2 might be involved in the degradation of viral RNA. To assess whether the observed effect is transferable to another cell type, we co-transfected PPP-RNA and capped-RNA into Hep3B cells or Hep3B cells lacking functional NUDT2, XRN1, or both. At 1 and $4 \mathrm{~h}$ post-transfection, NUDT2 depletion led to an increase in PPP-RNA stability as compared to the control (NTC) (Fig. 5d). Capped-RNA was not affected by NUDT2 depletion indicating specificity for degradation of PPP-RNA substrates. Importantly, depletion of XRN1 stabilized capped-RNA and PPP-RNA to a similar extent, underlining that XRN1 is in principle required to degrade both types of RNA. Similarly, cells co-depleted for NUDT2 and XRN1 showed increased PPP-RNA as well as capped-RNA levels. Notably, co-depletion did not result in synergistic effects compared to deleting single genes, further indicating that NUDT2 and XRN1 are operative in the same pathway (Fig. 5d). To further validate these findings, we infected Hep3B cells, depleted for NUDT2, XRN1, or NUDT2 and XRN1 with VSV-GFP (Fig. 5e). Both the lack of NUDT2 and XRN1 and the co-depletion led to a similar increase in GFP expression compared to the control, again pointing towards co-operative activities between NUDT2 and XRN1.

NUDT2 depletion in mice accelerates the growth of PPP-RNA generating viruses in vitro. We generated NUDT2 deficient mice from targeted embryonic stem cells (Supplementary Fig. 5a). Correct targeting of the Nudt2 locus was confirmed by PCR (Supplementary Fig. 5b). Homozygous knockout mice were viable, bred with expected Mendelian ratios (Supplementary Fig. 5c), and did not show an obvious phenotype under 

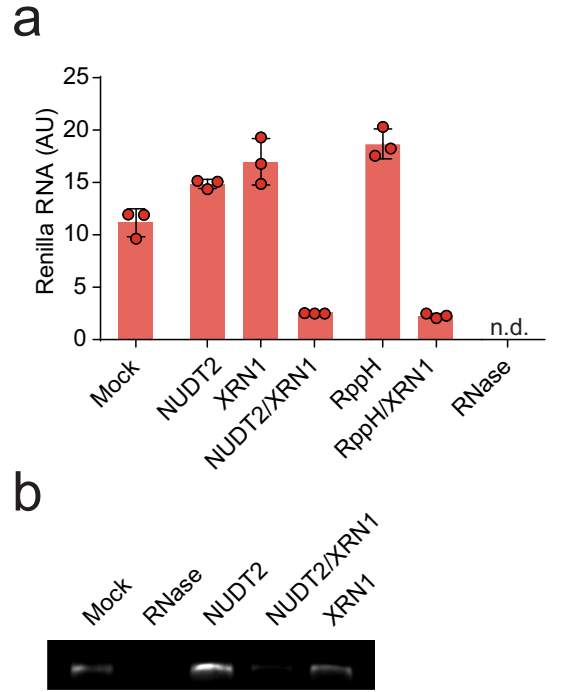

C

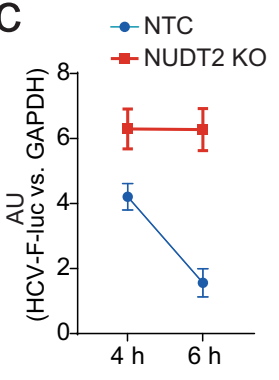

d

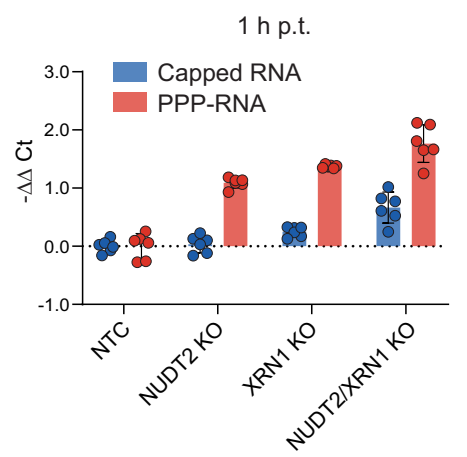

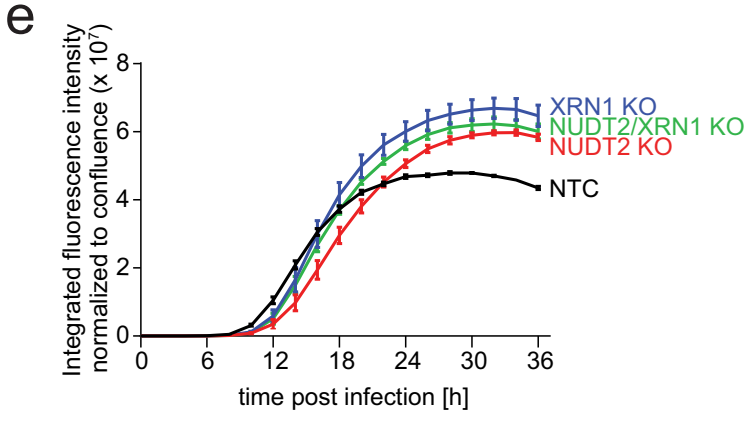

4 h p.t.

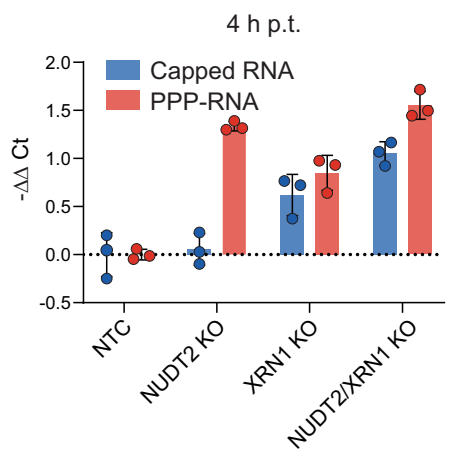

Fig. 5 NUDT2 is preparing RNA to serve as XRN1 substrate. a In vitro transcribed PPP-renilla RNA was left untreated (mock) or incubated with 600 nM NUDT2, 1 U XRN1, $600 \mathrm{nM}$ NUDT2 together with $1 \mathrm{U}$ XRN1, $5 \mathrm{U}$ RppH, or $5 \mathrm{U}$ RppH together with $1 \mathrm{U}$ XRN1 and $10 \mathrm{U}$ RNase for $4 \mathrm{~h}$. The remaining RNA was quantified by RT-qPCR. The bar plot shows the fold change compared to input RNA as the mean \pm SD of three technical replicates. $\mathbf{b}$ In vitro synthesized HCV RNA was treated with $5 \mathrm{U}$ RNase, $600 \mathrm{nM}$ NUDT2, 1 U XRN1, or co-treated with $600 \mathrm{nM}$ NUDT2 and $1 \mathrm{U}$ XRN1 and then analyzed on an agarose gel. c In vitro transcribed HCV RNA encoding luciferase was electroporated into HeLa CRISPR KO cells lacking NUDT2 or HeLa cells treated with a non-targeting-control vector (NTC). Viral RNA load was quantified 4 and $6 \mathrm{~h}$ post electroporation by the abundance of firefly luciferase RNA. d In vitro transcribed triphosphorylated renilla RNA and capped firefly luciferase RNA were co-electroporated into Hep3B CRISPR/Cas9 KO cells lacking NUDT2, $X R N 1$, or both NUDT2 and XRN1, or Hep3B cells treated with a non-targeting-control vector (NTC). RNA load was quantified 1 and $4 \mathrm{~h}$ post electroporation by quantifying the abundance of firefly luciferase or renilla RNA. e Hep3B treated with CRISPR/Cas9 lentivectors targeting NUDT2, XRN1, NUDT2, and XRN1 or with a non-targeting-control vector (NTC) were infected with VSV-GFP at MOI 0.01. GFP expression was monitored using automated live-cell microscopy. The data points are displayed as the mean of five technical replicates \pm SD. Shown is one representative experiment of three independent experiments.

non-infected conditions. To test whether NUDT2 deficiency may generally affect vulnerability to virus infections, we performed an unbiased proteome analysis of $\mathrm{Nudt2^{-/ }}$ and $\mathrm{Nudt} 2^{+/+}$bone marrow cells. This analysis allowed a parallel identification of 6288 proteins in total (Supplementary Table 2). As expected, NUDT2 could not be identified in the NUDT2 deficient mice (Fig. 6a). However, NUDT2 deficiency did not lead to significant differences in basal expression levels of the mouse proteome (considering 2866 proteins identified in 3 of 6 samples, FDR = $0.05, \mathrm{~S} 0=0.1$ ) (Supplementary Fig. 5d and Supplementary Table 2). Deficiency in the degradation of aberrant nucleic acids can lead to the accumulation of antiviral responses. However, compared to wild-type or heterozygous mice, $\mathrm{Nudt2^{-/ }}$ mice did not show spontaneous upregulation of mRNA encoding for classic antiviral proteins (e.g., Ifit3), inflammatory cytokines (e.g., IL-6), or U1 snRNA in heart, liver, lung, or spleen (Supplementary Fig. 5f) showing that NUDT2 depletion does not affect the basal immune status of mice. NUDT2 is known to cleave $\mathrm{Ap}_{4} \mathrm{~A}$ enzymatically, a dinucleotide generated under teratogenic conditions and during translational stress. To exclude that virus infection would elicit $\mathrm{Ap}_{4} \mathrm{~A}$ synthesis and that accumulation of this substance could be responsible for suppressing antiviral responses in cells, we tested the influence of $\mathrm{Ap}_{4} \mathrm{~A}$ on the growth of Semliki forest virus (SFV), a virus that is highly sensitive to antiviral activities of IFNs. Extracellular application of $\mathrm{Ap}_{4} \mathrm{~A}$ or its delivery into HeLa cells through lipofection impaired virus replication in a comparable manner as the double-stranded RNA analog polyI:C (Supplementary Fig. 5e), indicating that accumulation of $\mathrm{Ap}_{4} \mathrm{~A}$ is not responsible for increased virus growth. Collectively, NUDT2 depletion does not result in a general perturbation of the cellular innate immune response, and increased $\mathrm{Ap}_{4} \mathrm{~A}$ levels caused by NUDT2-deficiency do not promote virus growth. These observations are in line with a more direct effect of NUDT2 on viral RNA, i.e., the dephosphorylation of triphosphorylated RNA and its subsequent degradation.

We isolated total bone marrow (BM) from $N u d t 2^{-/-}$and littermate control mice and tested for replication of VSV wt and the IFN-stimulating variant VSV(M51A) (VSV-M2). Notably, accumulation of infectious virus particles was 10 - to 30 -fold increased in supernatants of bone marrow-derived from $\mathrm{Nudt} 2^{-/-}$ mice compared to corresponding $\mathrm{Nudt} 2^{+/+}$littermate controls (Fig. 6b). Similarly, compared to controls, MEFs from Nudt2-/animals showed up to a 100 -fold increase in VSV release into the supernatant and higher accumulation of VSV-N RNA (Fig. 6c, d). The increased virus growth also correlated with an increased virusinduced cytopathic effect in $\mathrm{Nudt2^{-/ }}$ compared to Nudt2+/+ MEFs (Fig. 6e). Importantly, infectious virus particles and viral RNA accumulation of HSV-1 (DNA virus), which does not generate PPP-RNA, were comparable in $\mathrm{Nudt2^{-/- }}$ and $\mathrm{Nudt2^{+/+ }}$ MEFs (Fig. 6f, g). Similarly, SFV (a virus predominantly sensed by 
a

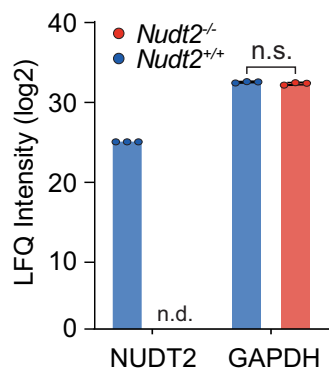

b

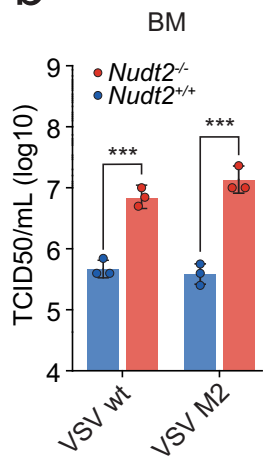

C

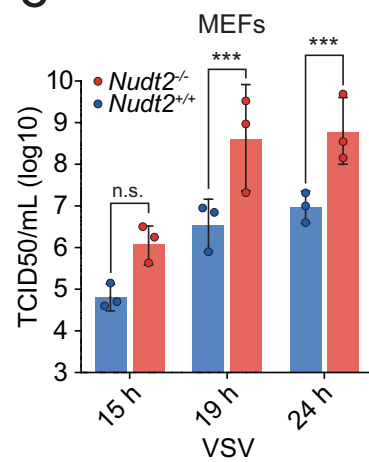

g

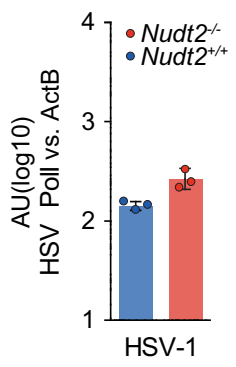

d

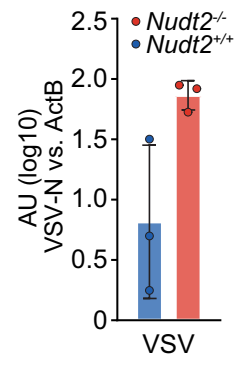

h

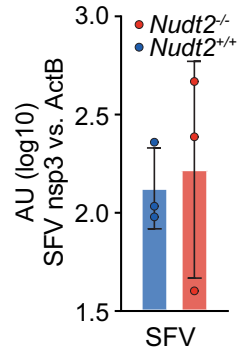

Fig. 6 Impact of NUDT2 on virus growth. a Bone marrow cells were isolated from Nudt2 knockout (Nudt2-/-), and control mice were tested for the abundance of NUDT2 by mass spectrometry. The mean LFQ intensities \pm SD for NUDT2 and GAPDH of six biological replicates are shown. A two-sided students t-test (FDR 0.05) was performed. b Accumulation of infectious virus particles in supernatants of Nudt2 $+/+$ or Nudt2 $/-$ bone marrow infected with either wild-type VSV, VSV-M2 at an MOI of 1 for $16 \mathrm{~h}$. The average TCID50/mL of three biological replicates \pm SD are shown. ${ }^{\star \star \star} p<0.001$ two-way ANOVA with Bonferroni's post-test. c Accumulation of VSV in supernatants of Nudt2 $2^{+/+}$and Nudt2-/- MEFs infected with VSV at an MOI of 0.001 for $16 \mathrm{~h}$. Average TCID $50 / \mathrm{mL}$ of three biological replicates \pm SD. ${ }^{\star \star \star} p<0.001$, two-way ANOVA with Bonferroni's post-test. $\mathbf{d}$ Viral RNA load in Nudt2 ${ }^{+/+}$or Nudt2 ${ }^{-/-}$MEFs infected with VSV. RNA levels were quantified by RT-qPCR analysis using specific primers for the VSV nucleoprotein transcript. Data were normalized to murine ActB RNA. The mean \pm SD of three biological repetitions are shown in arbitrary units. e Phase-contrast micrographs of Nudt2 $+/+$ or Nudt2-/- MEFs infected with VSV for $16 \mathrm{~h}$. The scale bars represent $100 \mu \mathrm{m}$. MEF cells of the indicated genotype were infected with HSV-1 (f, $\mathbf{g}$ ) or SFV (h) at an $\mathrm{MOI}$ of 2 for $16 \mathrm{~h}$. f Accumulation of infectious virus particles was analyzed. The graph shows the average TCID50/mL of three biological replicates \pm SD. $\mathbf{g}$, h Accumulation of viral RNA quantified by RT-qPCR analysis using specific primers for the DNA polymerase I of HSV or nsp3-transcript of SFV. Data were normalized to murine ActB RNA. Bar plots show average arbitrary units of three biological repeats \pm SD.

MDA5) ${ }^{23}$ replicated equally well in $\mathrm{Nudt} 2^{-/-}$compared to Nudt2 $2^{+/+}$MEFs (Fig. 6h). We concluded from these studies that mouse NUDT2 impairs the growth of PPP-RNA generating viruses in vitro, but it does not affect the accumulation of other viruses.

We proceeded to infect $\mathrm{Nudt2^{+/+ }}$ and $\mathrm{Nudt2^{-/- }}$ mice with VSV using an intranasal infection regime, causing encephalitis. Surprisingly, despite significant growth differences in cell culture experiments, the infection in vivo did not lead to significant differences in survival rates when comparing $N u d t 2^{+/+}$and $N u d t 2^{-/}$mice (Supplementary Fig. 6a). Moreover, the viral RNA load in the cerebellum of these mice was not significantly different. (Supplementary Fig. 6b) as well as transcripts for ISGs like Ifit3 or cytokines like IL-6 (Supplementary Fig. 5f). These experiments suggest that additional proteins with redundant functions are operative on an organismal level.

\section{Discussion}

RNA turnover is a highly conserved process and tightly controlled to regulate RNA abundance and to target erroneous RNAs for degradation. PPP-RNA, as present in viral genomes and to some extent in bacteria ${ }^{10}$, should, in principle, be protected from canonical cellular $5^{\prime}-3^{\prime}$ mRNA decay pathways present in higher eukaryotes. However, the half-life of PPP-RNA delivered into cells is only $\sim 0.5-1.0 \mathrm{~h}$, which is relatively short compared to the $9 \mathrm{~h}$ average half-life of cellular $\mathrm{mRNA}^{6,24}$. Although the PPPgroup protects PPP-RNA from XRN1-dependent degradation, depletion of this exonuclease increases viral RNA half-life ${ }^{25}$. This suggested the presence of a triphosphatase that converts PPPRNA into an XRN1 substrate ${ }^{4,26,27}$. Indeed, recently the phosphatases DUSP11 and DOM3Z (DXO) were identified as proteins that convert PPP-RNA into P-RNA, which serves as a substrate for XRN1 $1^{8,9,28}$. Since RNA degradation is an evolutionary, highly conserved process, we asked whether proteins known to be involved in PPP-RNA degradation in bacteria are also functionally active in mammalian cells. Using a siRNA screen, phosphate release assays, automated live-cell microscopy, and RT-qPCR, we identified that the protein NUDT2 could release phosphates from $5^{\prime}$-PPP RNA substrates and contributes to control the degradation of viral PPP-RNA. We show that NUDT2 is active on a broad range of PPP-RNA substrates but has a preference for processing longer substrates. NUDT2 sequentially releases two phosphates, and a monophosphorylated RNA is generated that can then serve as a substrate for the cellular exonuclease XRN1. This suggests that NUDT2 could be active in the degradation process of RNAs derived from diverse viruses. 
The Nudix hydrolase $\mathrm{RppH}$ has been shown to play a role in the dephosphorylation of $5^{\prime}$-PPP and mRNA degradation RNA in bacteria $^{11}$. Although PPP-mRNA is present in bacteria, recent reports of Luciano et al. suggest that $E$. coli predominantly contain diphosphorylated mRNA, which appears to be the preferred substrate of RppH in E. coliti ${ }^{10}$. The high structural similarity of $\mathrm{RppH}$ and mammalian NUDT2 indicates similar activities of both proteins, which is supported by the ability of NUDT2 and $\mathrm{RppH}$ to process phosphorylated RNA. Likely, NUDT2 has a preference for longer RNA constructs (compare Figs. 1b-e, 3a with Figs. 3c, $4 a-c)$ and thus can also act on viral diphosphorylated RNA, characteristic for some viruses, including reoviruses ${ }^{29}$, supported by the consecutive PPP-RNA dephosphorylation mechanism employed by NUDT2. Notably, NUDT2 is conserved throughout vertebrates, flies, worms, and bacteria, suggesting a broad functionality of this protein: Depending on the organism NUDT2 can be involved in physiological RNA turnover as well as antiviral defense mechanisms. NUDT2 deficiency in haploid KBM7 cells has been proposed to elicit an increased ISG expression pattern, which should result in reduced virus growth ${ }^{30}$. However, this reported phenotype does not match our observations in various diploid human and murine cells, the phenotype of Nudt2 knockout mouse, or the viral growth phenotype in Nudt2 depleted systems.

The importance of RNA degradation is highlighted by the fact that many viruses evolved mechanisms to escape RNA decay pathways and potentially hide from NUDT2 activity. Potential evasion strategies include capping of RNA by either employing the virus-derived capping machinery or seizing it from cellular mRNAs in a process called cap snatching 31,32 . Other RNA viruses, like viruses of the family Caliciviridae, protect their RNA by attaching a viral genome-linked protein ( $\mathrm{VPg})$. RNA of some viruses like Picornaviruses carry a structural element named internal ribosome entry site (IRES) that could also present a barrier for exonucleases like XRN1 ${ }^{33}$. HCV employs an unconventional mechanism to protect its RNA from degradation as it recruits the liver-specific microRNA miR-122 to its $5^{\prime}$-end. Through complementary binding to the HCV RNA, miR-122 protects the RNA from degradation by $\mathrm{XRN}^{6,34}$ by sterically hindering XRN1 binding to the $5^{\prime}$-end. Although NUDT2 dephosphorylated all tested RNA substrates, it may be that the activity of NUDT2 is similarly inhibited by steric hindrance under very special conditions, as seen for XRN-I. Flaviviruses form membranous replication factories that cover viral RNA from cellular proteins ${ }^{35}$, which may also be an evasion strategy to escape NUDT2 activity. It is not yet clear if NUDT2 can access such intracellular sub-compartments or if the activity of NUDT2 is limited to free $5^{\prime}$-PPP RNAs in the cytoplasm. Screening the impact of NUDT2 on more viruses would greatly enhance our understanding of the impact of NUDT2 dependent viral restriction and RNA decay. Since we could not observe a significant difference in survival rates between VSV-infected $\mathrm{Nudt2^{+/+ }}$ and $\mathrm{Nudt2^{-/ }}$ mice, we cannot exclude that other cellular pyrophosphatases have redundant functions. Such redundancies have also been reported for the activity of pattern recognition receptors (PRRs) in vivo. Despite the requirement of certain PRRs to induce cytokines and reduce virus growth in vitro, in vivo phenotypes were often less convincing. The RNA sensor Toll-like receptor (TLR) 7, for instance, only reveals its in vivo phenotype in the absence of the RIG-I sensing pathway ${ }^{36}$. Similarly, the in vivo activity of TLR3 strongly depends on the infection route of the pathogen ${ }^{37}$. Moreover, in an infection model for the DNA virus mouse Cytomegalovirus, the DNA sensor TLR9 only shows an effect in livers but not in spleens, clearly indicating an organspecific effect ${ }^{38}$. Similarly shown for PRRs, the in vivo phenotype of NUDT2 may only be visible when using sophisticated in vivo models or when specific infection models are used. On an organismal level, organ-specific expression differences or viral counter mechanisms could affect the impact of NUDT2.

Antiviral responses are often governed by proteins that are regulated by type-I IFNs. The expression of NUDT2 protein, however, does not change after cytokine treatment or virus infection. This may suggest that NUDT2, in addition to its ability to dephosphorylate viral RNA, may also have additional housekeeping functions. Interestingly, RNA Polymerase III products, including 5S ribosomal RNA, non-coding, and in some cases coding RNAs, should contain $5^{\prime}$-PPP termini. It is conceivable that proteins, such as NUDT2, DOM3Z (DXO), and DUSP11, are processing such aberrant RNAs or other RNA molecules to avoid stimulation of the innate immune system in the absence of pathogen encounter. Since self-RNA recognition may have dramatic effects on the cell, NUDT2, DUSP11, and DOM3Z (DXO) may fulfill redundant functions under steady-state conditions. Indeed, we could not obtain cells that lack both NUDT2 and DUSP11, despite the successful depletion of either of the two proteins. The full activity of these proteins may be required in case of viral infections since the abundance of viral PPP-RNA may exceed the catalytic capacity of either of these proteins.

\section{Methods}

Cells, reagents, and viruses. HeLa (ATCC CCL-2) and HEK293T (ATCC CRL3216) cells were described previously and were authenticated by ATCC ${ }^{39}$. Hep3B cells were obtained from Prof. R. Bartenschlager, University Hospital Heidelberg (Germany). Mouse embryonic fibroblasts (MEFs) were isolated from 13.5-day old embryos from heterozygous breeding pairs. Murine bone marrow was isolated from mouse femur, and tibia and cultured in DMEM supplemented with $10 \%$ fetal calf serum (FCS, GE Healthcare), $10 \mathrm{mM}$ HEPES pH 7.4, $1 \mathrm{mM}$ sodium pyruvate, and $2 \mathrm{mM} \mathrm{L}$-glutamine. Cell lines were maintained in DMEM (PAA Laboratories) containing $10 \%$ FCS and antibiotics $(100 \mathrm{U} / \mathrm{mL}$ penicillin, $100 \mu \mathrm{g} / \mathrm{mL}$ streptomycin). All viruses used are classified as BSL2 pathogens in Germany, and all experiments were carried out according to official regulations. Influenza A/SC35MNS1-2A-renilla-2A-NEP (IAV-ren) ${ }^{40}$, VSV, VSV-M2 ${ }^{41}$, VSV-GFP ${ }^{42}$, VSV-AV3$\mathrm{GFP}^{19}$ have been previously described. HSV-1 (F-strain) was from Soren Riis Paludan (Uni Aarhus, Denmark), VSV-Luc was a gift from Gert Zimmer (University Bern, Switzerland), and SFV was a gift from Andres Merits (University of Tartu, Estonia). Recombinant XRN1, CIP, and RppH and restriction enzymes for in vitro transcriptions were obtained from NEB, single nucleotides for in vitro transcriptions and phosphate release assays were from Jena Bioscience, RNasin was from Promega, TURBO DNase was obtained from Thermo Fisher Scientific, and T7 RNA polymerase was produced by the Core Facility of the MPI of Biochemistry. The CellTiter-Glo assay kit and the luciferase substrate d-Luciferin (E1602) were purchased from Promega. siRNAs were purchased from Qiagen or were synthesized by the Core Facility of the MPI of Biochemistry (see Supplementary Table 1).

Preparation of triphosphorylated or capped RNA. As RNA substrate, IVT4, a defined double-stranded triphosphorylated RIG-I ligand, was used as described ${ }^{43}$ To test the sequence specificity of NUDT2, RNA substrates with varying starting nucleotides, including single-stranded and double-stranded $5^{\prime}$-ends, were used as described ${ }^{13}$. In vitro transcribed RNA was generated from linear, double-stranded DNA templates, hybridized of complementary oligodeoxynucleotides (Supplementary Table 3). Except, the template for the generation of renilla RNA (Promega, E2231), which PCR amplified according to the manufacturer's instructions (2x HF Mastermix, NEB M0541S) with the corresponding oligodeoxynucleotides (Supplementary Table 3 ). The linear dsDNA product was purified (Monarch PCR Cleanup, T1030S) and directly used for in vitro transcription. RNA Sequences beginning with $5^{\prime}$-AG contained a T7 $\varphi 2.5$ promoter; all other templates contained a T7 46.5 promoter. The antisense strand of the DNA template was annealed with sense-oriented oligos covering the respective T7 promoter region ${ }^{44}$. These oligonucleotides were mixed 1:1 to a final concentration of $10 \mu \mathrm{M}$ each in $10 \mathrm{mM}$ Tris (pH 7.5-8.0), $50 \mathrm{mM} \mathrm{NaCl}$, and $1 \mathrm{mM} \mathrm{EDTA}$, heated to $95^{\circ} \mathrm{C}$ for $5 \mathrm{~min}$ and cooled down to $20^{\circ} \mathrm{C}$ at a rate of $0.1{ }^{\circ} \mathrm{C} / \mathrm{s}$ on a PCR cycler. For the preparation of renilla luciferase RNA, the pRL-SV40 plasmid was linearized by BamHI cleavage. To prepare firefly luciferase RNA, the RiboMAX Large Scale RNA Production Systems Kit (Promega) control plasmid was used. Transcription was carried out overnight at $37^{\circ} \mathrm{C}$ according to the manufacturer's protocol of the T7 RiboMAX Large Scale RNA Production Systems kit (Promega). After DNase treatment for $30 \mathrm{~min}$ at $37^{\circ} \mathrm{C}$ using $1 \mathrm{U}$ of RNase-free DNase per $\mu \mathrm{g}$ of DNA template, ivtRNA was purified using either the MinElute Cleanup kit (Qiagen) or the Monarch RNA Cleanup Kit (NEB).

For the preparation of HCV PPP-RNA, the pFK-JFH plasmid DNA ${ }^{45}$ was linearized by MluI cleavage. Transcription was carried out for $6-8 \mathrm{~h}$ at $37^{\circ} \mathrm{C}$ in 
$5 \times$ transcription buffer (200 mM Tris $\mathrm{pH} 8,30 \mathrm{mM} \mathrm{MgCl}_{2}, 10 \mathrm{mM}$ Spermidine, and $5 \mathrm{mM}$ DTT), $2.5 \mathrm{mM}$ ATP, $2.5 \mathrm{mM}$ UTP, $2.5 \mathrm{mM}$ CTP, $2.5 \mathrm{mM}$ GTP, RNasin (Promega), and $1000 \mathrm{U}$ T7 RNA polymerase (Core Facility of the MPI of Biochemistry), in $100 \mu \mathrm{L}$ final volume. $2 \mu \mathrm{L}$ TURBO DNase (Thermo Fisher Scientific) was added for $30 \mathrm{~min}$ at $37^{\circ} \mathrm{C}$. Reactions were purified with mini Quick Spin RNA Columns (Roche) according to the manufacturer's protocol. For the 44mer single-stranded RNA, the pGEX-6P-T7-44-mer-RNA ${ }^{46}$ plasmid linearized by EcoRI cleavage served as a template. RNA was synthesized by in vitro transcription according to the MEGAscript T7 Kit (Thermo Fisher Scientific). After incubation for $16 \mathrm{~h}$ at $37^{\circ} \mathrm{C}$ and TURBO DNase (Thermo Fisher Scientific) treatment for $30 \mathrm{~min}$ at $37^{\circ} \mathrm{C}$, the RNA was purified according to the Direct-zol RNA extraction kit (Zymo Research). For the generation of double-stranded RNA, a 20-nt antisense RNA (Metabion) (5'-acucucucucucucucuccc- $\left.3^{\prime}\right)$ was annealed to the in vitrotranscribed RNA. In vitro transcribed RNA was capped using the ScriptCap Cap 1 Capping System (CellScript, C-SCCS1710).

siRNA-mediated knockdown experiments. Duplex siRNAs were transfected using either siPrime transfection reagent (GE Healthcare) for the Nudix hydrolase siRNA screen or Metafectine Pro transfection reagent with the $\mathrm{SI}^{+}$Buffer (Biontex) for targeted gene knockdowns. Transfection was performed according to the manufacturer's instructions for HeLa cells. Briefly, we transfected $15 \mathrm{pmol}$ of pooled siRNAs per $1 \mathrm{e} 5$ cells in a 24 well format. Cells were infected $48 \mathrm{~h}$ after siRNA knockdown. Duplex siRNAs were either purchased from Qiagen or synthesized by the Core Facility at the MPI of Biochemistry (Supplementary Table 1). Cell viability after siRNA-mediated knockdown was assessed with the CellTiter-Glo (Promega) luminescent cell viability assay, according to the manufacturer's protocol.

Virus infections and determination of virus titers and growth. To determine the impact of NUDT2 knockout on virus growth, MEFs and bone marrow were seeded the day before infection. Cells were infected with VSV wt at a multiplicity of infection (MOI) of 0.001 , SFV at an MOI of 0.001 , HSV-1 at an MOI of 2, VSVLuc at an MOI of 0.001, IAV-renilla at an MOI of 0.1, and HSV-Luc at an MOI of 2. Bone marrow was infected with VSV wt and VSV M2 at an MOI of 1. Twentyfour hours post-infection or as indicated specifically in the figure legend, cells were harvested for quantitative RT-qPCR, Western Blot or and the supernatant was harvested to test for virus accumulation by $50 \%$ tissue culture-infective dose (TCID50) assays on Vero E6 cells. To quantify the effects of siRNA-mediated knockdown of Nudix hydrolases, cells were infected $48 \mathrm{~h}$ after the knockdown was performed with VSV-Luc at an MOI of 0.001 for $24 \mathrm{~h}$, IAV-renilla at an MOI of 1 for $48 \mathrm{~h}$, VSV-GFP at an MOI of 0.001 for $24 \mathrm{~h}$, and VSV-M2-GFP at an MOI of 0.0001 for $24 \mathrm{~h}$. GFP levels were determined fluorometrically. $Z$-scores were calculated from three independent experiments individually for every gene and virus, as previously described ${ }^{47}$.

For RT-qPCR analysis, RNA was isolated using the NucleoSpin RNA Plus kit (Macherey Nagel) according to the manufacturer's protocol. For proteomic analysis, cell pellets were snap-frozen in liquid nitrogen before further processing. For western blot analysis, cells were lysed in Laemmli buffer, boiled for $10 \mathrm{~min}$ at $95^{\circ} \mathrm{C}$, and subjected to SDS-polyacrylamide gel electrophoresis and western blot analysis. For GFP-tagged viruses, virus growth was measured by determining GFP levels in a microplate reader (Tecan Infinite 200 Pro) with an excitation wavelength of $485 \mathrm{~nm}$ and an emission wavelength of $535 \mathrm{~nm}$. For luciferase-tagged viruses, cells were lysed in Passive Lysis Buffer (Promega), and virus load was determined by measuring firefly or renilla-luciferase. VSV-GFP growth kinetics were monitored and analyzed using the IncuCyte S3 Live-cell imaging system and software (Essen Bioscience). Heb3b cells, transduced with a non-targeting control vector or with a CRISPR/Cas9-mediated depletion of either NUDT2, XRN1, or NUDT2 and XRN1, were infected with VSV-GFP at an MOI of 0.01 in five technical replicates. For each technical replicate, two phase-contrast and green fluorescence images were acquired by a $10 \times$ objective. Fluorescence images were further processed to remove the background signal using a top-hat filter. The integrated intensity of GFP+ cells per image was then normalized to the total area of cells by division for each time point.

Protein expression and purification. DNA sequences of NUDT2, NUDT2 E58A, NUDT12, NUDT12 E369A, NUDT14, NUDT14 E121A, NUDT17, NUDT17 E124A were obtained as gBlocks from IDT containing overhangs suitable for SLIC cloning. All constructs were cloned into pCoofy4, providing an $N$-terminal His6-MBP tag. Protein expression and purification were performed by the Core Facility of the MPI of Biochemistry. Briefly, plasmids were transformed in BL21-AI pRARE bacteria, and protein expression was carried out in an autoinduction medium containing $0.2 \%$ arabinose. Cells were lysed using an AVESTIN high-pressure homogenizer, and cleared lysate was used for protein purification using a HisTrap HP column (GE Healthcare: 17-5247-01) and further purified by gel filtration using a Superose 6 PC 3.2/30 (GE Healthcare: 17-0673-01) (mobile phase: $20 \mathrm{mM}$ Tris- $\mathrm{HCl} \mathrm{pH} 7.5$, $250 \mathrm{mM} \mathrm{NaCl}, 10 \mathrm{mM} \mathrm{MgCl}$ ). Proteins were dialyzed with D-Tube Dialyser Midi

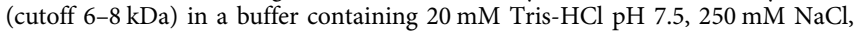
$10 \mathrm{mM} \mathrm{MgCl}_{2}$, and $1 \mathrm{mM} \mathrm{DTT}$. The identity of recombinant proteins was confirmed by mass spectrometry.
To test phosphate release from mammalian expressed NUDT2 cells, N-terminally 6-myc-tagged NUDT2, NUDT2 E58A, and NS1 of Influenza A virus (with mutated RNA binding domain (R38A, K41A) of strain A/PR8/34) were transiently expressed from pCS2-N-6xMyc plasmids. For this, HEK293T cells were transfected using $1.3 \mu \mathrm{g}$ of plasmid per $1 \mathrm{e} 6$ cells and a ratio of the transfection reagent PEI to plasmid DNA of 3:1. The cells were collected after two days, washed $3 \times$ with PBS, pelleted, and frozen. Subsequently, the cells were lysed with TAP lysis buffer (50 mM Tris- $\mathrm{HCl}$ at $\mathrm{pH} 7.5,5 \%$ glycerol, $0.2 \% \mathrm{NP}-40,1.5 \mathrm{mM} \mathrm{MgCl} 2$, $100 \mathrm{mM} \mathrm{NaCl}$, supplemented with complete protease and phosphatase inhibitor cocktails (Roche)) on ice for $30 \mathrm{~min}$, followed by sonication $\left(10 \mathrm{~min}, 4^{\circ} \mathrm{C}\right)$ and clearing of the lysate $\left(4000 \mathrm{~g}, 30 \mathrm{~min}, 4^{\circ} \mathrm{C}\right)$. The expressed, Myc-tagged proteins were then precipitated using $80 \mu \mathrm{L}$ slurry of equilibrated $\alpha$-myc agarose beads (Chromotek, yta-20). Beads were washed three times with NEB 2 reaction buffer to equilibrate for the phosphate release assay.

SDS-PAGE and western blotting. Validation of precipitation of myc-tagged proteins: Immunoprecipitated proteins were eluted in the presence of 1x SDS sample buffer (62.5 mM Tris- $\mathrm{HCl}, 2 \%$ SDS, $10 \%$ glycerol, $50 \mathrm{mM}$ DTT, $0.01 \%$ bromophenol blue, $\mathrm{pH} 6.8$ ) and by boiling the samples at $95^{\circ} \mathrm{C}$ for $5 \mathrm{~min}$. Eluted proteins were then separated on a $4-12 \%$ Bis-Tris acrylamide gel (NuPAGE, Invitrogen) at $100 \mathrm{~V}$ for $90 \mathrm{~min}$ and transferred onto a $0.2 \mu \mathrm{m}$ nitrocellulose membrane by wet-blotting at $300 \mathrm{~mA}$ for $1 \mathrm{~h}$. The membrane was then blocked with $5 \%$ milk, $3 \times$ washed with PBS with $0.25 \%$ Tween-20 (PBS-T) for 5 min each, and stained with primary antibodies in $5 \%$ milk followed by incubation with an appropriate secondary antibody.

Primary antibodies used in this study were as follows: monoclonal mouse $\alpha$ NUDT2 (Santa Cruz: sc-271410, 1:1000 dilution), monoclonal mouse $\alpha$ - $\beta$-ActinHRP (Santa Cruz: sc-47778, 1:1000 dilution), monoclonal mouse $\alpha$-myc-HRP (Roche, 11814150001, 1:2000 dilution), and secondary horseradish peroxidase (HRP)-coupled antibody rabbit $\alpha$-mouse IgG (Cell Signaling, 7076, 1:2000 dilution).

Phosphate release assay. For time-resolved analysis, the phosphatase activities of Nudix hydrolases, CIP, or RppH were analyzed with the EnzChek Phosphate Assay (E6646, Sigma-Aldrich) according to the manufacturer's instructions. For optimal performance, the following adjustments were made: one replicate $(200 \mu \mathrm{L}$ reaction volume) included $600 \mathrm{nM}$ NUDT protein or the corresponding mutant, $4.5 \mu \mathrm{g}$ of RNA substrate, $0.5 \mathrm{U}$ of PNP, $0.2 \mathrm{mM} \mathrm{MESG}$, and NEB 2.0 buffer. This setting was validated to yield optimal assay performance (Supplementary Fig. 3a). The absorbance was measured at $360 \mathrm{~nm}$ every three min. The phosphate release was calculated from a linear standard curve specific for each time point.

For single time-points, the ability of Nudix hydrolases to release phosphates from RNA was tested with in vitro transcribed RNA (ivtRNA), and ribonucleotide triphosphate (rNTPs). The malachite green assay was performed according to the manufacturer's instructions (MAK307, Sigma-Aldrich) after incubating $900 \mathrm{ng}$ IVT4 with NUDT2 or NUDT2 E58A in NEB2 buffer for $3 \mathrm{~h}$ at $37^{\circ} \mathrm{C}$. This setting was validated to yield optimal assay performance. In order to test NUDT2 activity on a panel of possible substrates, NUDT2 $(600 \mathrm{nM})$ and NUDT2 E58A $(600 \mathrm{nM})$ were incubated for $3 \mathrm{~h}$ with rATP, rGTP, rUTP, and $\mathrm{rCTP}(2.5 \mu \mathrm{M})$ for $3 \mathrm{~h}$ at $37^{\circ} \mathrm{C}$ in NEB2 reaction buffer. CIP (5 U) (NEB) was used as a positive control for released inorganic phosphate in all experiments. For immunoprecipitated NUDT2, NUDT2 E58A or NS1 (IAV) derived from HEK cell lysate, $20 \mu \mathrm{L}$ of beads were incubated in $40 \mu \mathrm{L}$ NEB 2 reaction buffer, including $900 \mathrm{ng}$ IVT4, for $3 \mathrm{~h}$ at $37^{\circ} \mathrm{C}$. Beads were separated $(500 \mathrm{~g}, 1 \mathrm{~min}, \mathrm{RT})$, and the phosphate release assay was performed using the malachite green assay as described above.

Phosphate release from $\boldsymbol{\gamma}$-32P-labeled RNA. $\gamma^{-32} \mathrm{P}$ labeled RNA was synthesized by in vitro transcription according to the MEGAscript T7 Kit (Thermo Fisher Scientific) supplemented with $0.17 \mu \mathrm{M}\left[\gamma^{-32} \mathrm{P}\right]$ GTP (Perkin Elmer). For the 44-mer single-stranded RNA, the pGEX-6P-T7-44mer-RNA ${ }^{46}$ plasmid linearized by EcoRI cleavage served as a template. For the dsRNA IVT4, two annealed primer pairs (Supplementary Table 3$)^{43}$ were used as a template. After incubation for $16 \mathrm{~h}$ at $37^{\circ} \mathrm{C}$ and DNase digestion with TURBO DNase (Thermo Fisher Scientific) for $30 \mathrm{~min}$ at $37^{\circ} \mathrm{C}$, the RNA was purified according to the Direct-zol RNA extraction kit (Zymo Research). For the generation of a double-stranded RNA of the 44-mer ssRNA, a 20-nt antisense RNA (Metabion) (5'-acucucucucucucucuccc- $\left.3^{\prime}\right)$ was annealed to the in vitro transcribed RNA.

The labeled RNAs were incubated with purified NUDT2 $(600 \mathrm{nM})$, NUDT12 (600 nM), NUDT14 (600 nM), NUDT17 (600 nM), and RppH (5 U) purchased from NEB in a solution $(20 \mu \mathrm{L})$ containing NEB2 buffer at $37^{\circ} \mathrm{C}$. Reaction samples $(10 \mu \mathrm{L})$ were taken at 0 and $90 \mathrm{~min}$ and quenched with $2.5 \mu \mathrm{L}$ of EDTA $(100 \mathrm{mM}$, pH 8.0), analyzed by TLC on PEI-cellulose (Merck Millipore), and developed with potassium phosphate buffer $(0.3 \mathrm{M}, \mathrm{pH} 7.5)$. Spot intensities were imaged by using a GE Typhoon FLA 9000 Imager. For time-course experiments, the labeled RNA was incubated with purified NUDT2 or NUDT2 E58A $(600 \mathrm{nM})$ in a solution $(50 \mu \mathrm{L})$ containing NEB2 Buffer for $0-90 \mathrm{~min}$ at $37^{\circ} \mathrm{C}$. Reaction samples $(10 \mu \mathrm{L})$ were quenched at time intervals with $2.5 \mu \mathrm{L}$ of EDTA $(100 \mathrm{mM}, \mathrm{pH} 8.0)$ and analyzed by TLC on PEI-cellulose (Merck Millipore) developed with potassium 
phosphate buffer $(0.3 \mathrm{M}, \mathrm{pH}$ 7.5). Spot intensities were imaged by using a GE Typhoon FLA 9000 Imager.

Synthesis of pppApG and pppGpA. Dinucleotides were synthesized using DMT$2^{\prime}$-O-TBDMS-rA(bz), DMT-2'-O-TBMDS-rG(ib) amidites, and $\mathrm{rA}(\mathrm{Bz})$ and rG(ibu) CPG (Chemgenes) on a $10 \mu \mathrm{M}$ scale by using standard solid-phase oligoribonucleotide coupling techniques. The CPG-bound dimers were triphosphorylated using the cyclotriphosphate protocol of triphosphate synthesis ${ }^{48}$, except that after deprotection of the dinucleotides, the reaction mixture was converted into the triethylammonium salt using Dowex Et3NH+ before reverse-phase chromatography on a Source RP column. DecNHpppApG and DecNHpppGpA RP-HPLC product peaks were converted into pppApG and pppGpA as described. The pure pppGpA and pppApG were isolated as triethylammonium salts by ionexchange chromatography on a Hi Screen DEAE FF column using a gradient of $0.0-0.6 \mathrm{M}$ Triethylammonium bicarbonate. The integrity of the pppApG and pppGpA dimers was verified by LC-MS analysis.

HPLC analysis. A short RNA (pppGpA, 0.1 OD260) was incubated for $2 \mathrm{~h}$ at $37^{\circ} \mathrm{C}$ in a solution $(15 \mu \mathrm{L})$ containing NEB2 buffer $(1 \times)$ with recombinant NUDT2 $(600 \mathrm{nM})$, NUDT2 E58A (600 nM), RppH (5 U) or left untreated. Reactions mixtures were quenched with $3.75 \mu \mathrm{L}$ of EDTA $(100 \mathrm{mM}, \mathrm{pH} 8.0)$ and diluted 1:20 with water. For time-course measurements, the two short RNAs (pppGpA or pppApG, 0.1 OD260 each) were incubated with purified recombinant NUDT2 (600 nM), NUDT14 (600 nM), and NUDT2 E58A (600 nM), respectively, in a solution $(75 \mu \mathrm{L})$ containing NEB2 buffer $(1 \times)$ at $37^{\circ} \mathrm{C}$. Reaction samples $(15 \mu \mathrm{L})$ were taken after $0,1,3,6$, and $19 \mathrm{~h}$, quenched with $3.75 \mu \mathrm{L}$ of EDTA $(100 \mathrm{mM}, \mathrm{pH}$ $8.0)$, and diluted 1:20 with water. Aliquots $(80 \mu \mathrm{L})$ were analyzed by reversed-phase (RP)-HPLC on an AGILENT 1100 System using an XBridge C18 column $(3.5 \mu \mathrm{m}$, $150 \times 2.1 \mathrm{~mm}$, WATERS). Chromatography was performed using a two-eluent buffer system. Buffer A consists of an aqueous solution of $100 \mathrm{mM}$ Et3NHOAc, $\mathrm{pH}$ 7.8, and buffer B consists of an aqueous solution of $100 \mathrm{mM} \mathrm{Et3NHOAc,} \mathrm{pH} \mathrm{7.8,}$ and $40 \%(\mathrm{v} / \mathrm{v}) \mathrm{MeCN}$. Chromatograms were recorded at a wavelength of $260 \mathrm{~nm}$. HPLC was performed using the following gradient condition: $0 \% \mathrm{~B}$ over $4 \mathrm{~min}$, to $15 \%$ B over $20 \mathrm{~min}$, to $18 \% \mathrm{~B}$ over $16 \mathrm{~min}$ to $100 \% \mathrm{~B}$ over $10 \mathrm{~min}$, held at $100 \% \mathrm{~B}$ for $15 \mathrm{~min}$, to $0 \% \mathrm{~B}$ over $2 \mathrm{~min}$, held at $0 \% \mathrm{~B}$ for $13 \mathrm{~min}$ with a flow rate of $0.3 \mathrm{~mL} /$ min. Quantification of eluted compounds was performed by integration of the corresponding peak areas.

Analysis of RNA integrity and transcript level analysis. To assess RNA integrity after treatment with recombinant Nudix hydrolases, small reaction samples were analyzed by the Bioanalyzer (Agilent) using the Agilent Small RNA kit according to the manufacturer's protocol. For quantitative RT-qPCR analysis, total RNA from cells was isolated using the NucleoSpin RNA Plus kit (Machery-Nagel). RNA from mouse cerebellum, heart, liver, lung, or spleen was extracted using Lysing Matrix M and the FastPrep-24 (MP Biomedicals) instrument. Organs were directly lysed in LBP buffer, and RNA isolation was continued using the NucleoSpin RNA Plus kit (Machery-Nagel). Next, 200-500 ng of RNA was reverse-transcribed with PrimeScript RT Master Mix (TAKARA) and after that quantified by RT-qPCR using the QuantiFast SYBR Green RT-PCR Kit (Qiagen) and a CFX96 Touch Real-Time PCR Detection System (Bio-Rad). Each cycle included $10 \mathrm{~s}$ at $95{ }^{\circ} \mathrm{C}$ and $30 \mathrm{~s}$ at $60^{\circ} \mathrm{C}$, followed by a melting curve analysis. Primer sequences are depicted in Supplementary Table 4 .

Generation of CRISPR/Cas9 knockout cells. Double-stranded 30-nt guide sequences (Supplementary Table 5) targeting the human NUDT2 gene and a nontargeting control were designed and cloned into pLentiCRISPRv2 (Addgene $\# 52961)$ as previously described ${ }^{49,50}$. HEK 293 T cells were co-transfected with the obtained plentiCRISPR v2 vector $(6 \mu \mathrm{g})$, pxPAX2 $(3 \mu \mathrm{g})$, and pMD2G $(1.5 \mu \mathrm{g})$ in a $10 \mathrm{~cm}$ dish using PEI. $24 \mathrm{~h}$ post-transfection, the medium was replaced with $10 \mathrm{~mL}$ of fresh DMEM. Seventy-two hours post-transfection, the medium was collected, filtered through a $0.45 \mu \mathrm{m}$ pore size membrane (Millipore), and HeLa cells were infected with the lentivirus. Twenty-four hours post-infection, the medium was replaced with DMEM containing puromycin $(1 \mu \mathrm{g} / \mathrm{mL})$ for four passages. Knockout efficiency was analyzed by western blotting.

RNA degradation assays. Degradation of $1000 \mathrm{ng}$ of an in vitro transcribed renilla or HCV PPP-RNA carrying a firefly luciferase was assessed by incubating it either with 10 U RNase A, $600 \mathrm{nM}$ recombinant NUDT2, 1 U recombinant XRN1, $5 \mathrm{U}$ recombinant RppH, or both $600 \mathrm{nM}$ NUDT2 and $1 \mathrm{U}$ XRN1 or $5 \mathrm{U}$ RppH and $1 \mathrm{U} \mathrm{XRN1}$ for $4 \mathrm{~h}$ at $37^{\circ} \mathrm{C}$. To determine RNA levels after incubation, renilla RNA was quantified using RT-qPCR, and the presence of HCV PPP-RNA was assessed using $1 \%$ agarose gels. The stability of in vitro transcribed HCV PPP-RNA encoding luciferase was determined by electroporation into HeLa CRISPR/Cas9 KO cells lacking NUDT2 or HeLa cells treated with a non-targeting control vector. HCV PPP-RNA load was quantified 4 and $6 \mathrm{~h}$ post electroporation by quantifying the abundance of firefly luciferase RNA by RT-qPCR. To determine degradation of capped or PPP-RNA, capped firefly luciferase and PPP-renilla luciferase were mixed in equimolar amounts and were co-electroporated into Hep3B CRISPR/ Cas9 KO cells lacking NUDT2, XRN1, or both NUDT2 and XRN1, or Hep3B cells treated with a non-targeting-control vector. Cells were incubated for 1 and $4 \mathrm{~h}$ at $37^{\circ} \mathrm{C}$. To determine RNA levels after incubation, renilla or firefly luciferase RNA was quantified using RT-qPCR and normalized to RPLP0 RNA.

Ap4A treatment. HeLa cells were transfected with $1 \mu \mathrm{g}$ poly(I:C) using $1 \mu \mathrm{L}$ METAFECTENE Pro or $250 \mathrm{nmol} \mathrm{Ap}{ }_{4} \mathrm{~A}$ using $2 \mu \mathrm{L}$ METAFECTENE Pro or $250 \mathrm{nmol}$ of $\mathrm{Ap}_{4} \mathrm{~A}$ was added directly to the media ${ }^{51,52}$. As controls, the cells were stimulated with PBS in combination with and without METAFECTENE Pro. Twenty-four hours post-treatment, the cells were infected with SFV6-2SG NanoLuc at an MOI of 0.0035 or left uninfected for $24 \mathrm{~h}$. The cells were lysed in Passive Lysis Buffer, and virus growth was assessed by measurement of renilla luciferase.

Generation of Nudt2 knockout mice. The Nudt2 allele was rendered unfunctional by introducing a poly(A) signal after exon 1 in a construct purchased from the European Conditional Mouse Mutagenesis consortium (EUCOMM). This genetically modified ES cell clone (Clone ID: EPD0146_2_H06, Cell type: JM8.N4) was injected into C57BL/6 blastocyst donors. Male chimeras were bred with C57BL/6

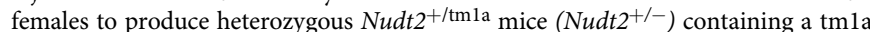
cassette encoding for the lacZ gene, a neomycin resistance, a splice acceptor, and a polyA site flanked by FRT sites. Heterozygous animals were then bred to generate homozygous Nudt2 $2^{\text {tmla/tmla }}$ mice (Nudt $2^{-/}$).

For genotyping NUDT2 knockout mice, ear punches were collected from 21 to 23-days old mice and genotyped by PCR using the following primers: Nudt2_fwd: $5^{\prime}$-ccagctttcttgtacaaagtgg-3', Nudt2 wt_fwd: 5'-gaatttctgctgctgcaggc-3', Nudt2_rev: $5^{\prime}$-cctagtgaagggacaaagcagc- $3^{\prime}$ and using the following parameters: $94^{\circ} \mathrm{C}$ for $1 \mathrm{~min}$, followed by 35 cycles of $94^{\circ} \mathrm{C}$ for $30 \mathrm{~s}, 65^{\circ} \mathrm{C}$ for $20 \mathrm{~s}$, and $72^{\circ} \mathrm{C}$ for $1 \mathrm{~min}$. The wild-type allele was detected as a band at $492 \mathrm{bp}$, whereas the inserted tmla cassette was detected as bands of 417 and $912 \mathrm{bp}$.

Ethics statement and mouse infections. All animal experiments were performed in compliance with the German Animal Welfare Law (TierSchG BGBl. S. 1105; 25.05.1998). The mice were housed and handled in accordance with good animal practice as defined by FELASA. All animal experiments were approved by the responsible state office (Landesverwaltungsamt Sachsen-Anhalt), the University of Magdeburg, under permit number AZ 42502-2-1344. During intranasal infections (i.n), 8-12-week-old mice were first anesthetized by i.p. injection with a mixture of ketamine $(100 \mathrm{mg} / \mathrm{g}$ body weight $)$ and xylazine $(5 \mathrm{mg} / \mathrm{g}$ body weight). They then were infected with $5 \mathrm{e} 6$ plaque-forming units (PFU) of VSV in $20 \mu \mathrm{L}$ PBS unless otherwise indicated. Mice that lost more than $20 \%$ of their body weight were sacrificed.

Proteomic analysis of Nudt2 knockout in mouse bone marrow cells. For proteomic analysis, wild-type and Nudt2 knockout bone marrow cells (5e6/cell line/ replicate; 4 replicates) were lysed in $300 \mu \mathrm{L}$ of lysis buffer (4\% SDS, $10 \mathrm{mM}$ DTT in $50 \mathrm{mM}$ Tris pH 7.6) supplemented with complete protease inhibitor (Roche) and boiled for $10 \mathrm{~min}$ at $95^{\circ} \mathrm{C}$. The protein concentration of cleared cell lysates was assessed using Pierce $660 \mathrm{~nm}$ Protein Assay (Thermo Fisher Scientific) according to the manufacturer's protocol. Protein concentrations for all cell lines and replicates were equalized to $35 \mu \mathrm{g}$ protein content and alkylated with $55 \mathrm{mM}$ IAA for $20 \mathrm{~min}$ in the dark. Proteins were precipitated with acetone and resuspended in $150 \mu \mathrm{L}$ of denaturation buffer (6 M Urea, $2 \mathrm{M}$ Thiourea in $10 \mathrm{mM}$ HEPES $\mathrm{pH}$ 8.0). Protein digestion was performed by adding 1:100 (protein:enzyme) trypsin and LysC overnight at room temperature. Peptides were purified on stage tips and analyzed by LC-MS/MS using the EASY-nLC 1200 system coupled to a Q Exactive HF mass spectrometer (Thermo Fisher Scientific). Peptide mixtures were separated on a $50 \mathrm{~cm}$ C18-reversed-phase column (Reprosil-Pur $120 \mathrm{C} 18-\mathrm{AQ}, 1.9 \mu \mathrm{M}$, $200 \times 0.075 \mathrm{~mm}$; Dr. Maisch) using a 180 -minute linear gradient of $5-30 \%$ buffer B $(0.1 \%$ formic acid and $80 \%$ ACN $)$ with a flow rate of $250 \mathrm{~nL} / \mathrm{min}$ The mass spectrometer was set up to run a Top10 method acquiring full scans $(300-1,600 \mathrm{~m} / \mathrm{z}, R=60,000$ at $200 \mathrm{~m} / \mathrm{z})$ at a target of $3 \mathrm{e} 6$ ions, followed by isolation of the ten most abundant ions, HCD fragmentation (target 1e5 ions, maximum injection time $120 \mathrm{~ms}$, isolation window $1.4 \mathrm{~m} / \mathrm{z}$, NCE $27 \%$, and underfill ratio of $20 \%)$ and detection in the Orbitrap analyzer.

Raw data processing and statistical analysis. Peptide identification and quantification were performed using MaxQuant (version 1.6.17.0). For all MaxQuant searches, typical default parameters were employed. Spectra were searched against forward and reverse sequences of the reviewed mouse proteome, including isoforms (Uniprot, UP000000589). Settings included carbamidomethylation of cysteine as fixed modification and oxidation of methionine and $\mathrm{N}$-terminal protein acetylation as variable modifications. Trypsin/P was specified as the proteolytic enzyme. The minimal peptide length was defined as 7 amino acids, and matchbetween-run was disabled for LFQ determination.

The protein groups were further analyzed with Perseus (1.6.14.0). The matrix included 6288 proteins and was filtered with the default settings: potential contaminant only identified by site and reverse. After $\log 2$ transformation, a twosided students $t$-test (FDR 0.05) was performed, including proteins with at least three valid values (in total) and imputed (width 0.3 and downshift 1.8) 
Phylogenetic analysis. To establish the phylogenetic relationship between different human Nudix hydrolases and bacterial $\mathrm{RppH}$, protein sequences from all human Nudix hydrolases and E. coli RppH were analyzed using MAFFT ${ }^{53}$ with standard settings. The resulting tree was displayed using Dendroscope ${ }^{54}$ and visually adapted using Adobe Illustrator CS6 (16.0.3).

Multiple sequence alignment. Protein sequences of NUDT2 from Homo sapiens, Danio rerio, Caenorhabditis elegans, Mus musculus, Xenopus laevis, Gallus gallus, and Drosophila melanogaster were collected and aligned using the Clustal Omega tool with standard settings. The multiple sequence alignment was visualized using ESPript $3.0^{55}$.

Statistical analysis. All data were analyzed either using GraphPad Prism (8.4.3) or R (4.0.2) using R Studio (1.3.1056). Data acquired with the Incucyte live-cell imaging platform were analyzed using IncuCyte Analysis Software (2019B Rev2).

Reporting summary. Further information on research design is available in the Nature Research Reporting Summary linked to this article.

\section{Data availability}

The data supporting the findings of this study are available from the corresponding authors upon reasonable request. Source data for the figures and supplementary figures are provided as a Source Data file. The LC-MS/MS data and MaxQuant output generated in this study have been deposited in the ProteomeXchange Consortium via the PRIDE partner repository ${ }^{56}$ under accession code P9. Source data are provided with this paper.

Received: 10 May 2020; Accepted: 8 November 2021;

Published online: 25 November 2021

\section{References}

1. Arribas-Layton, M., Wu, D., Lykke-Andersen, J. \& Song, H. Structural and functional control of the eukaryotic mRNA decapping machinery. Biochim. Biophys. Acta 1829, 580-589 (2013).

2. Pellegrini, O., Mathy, N., Condon, C. \& Benard, L. In vitro assays of 5' to 3'exoribonuclease activity. Methods Enzymol. 448, 167-183 (2008).

3. Gebhardt, A., Laudenbach, B. T. \& Pichlmair, A. Discrimination of self and non-self ribonucleic acids. J. Interferon Cytokine Res. 37, 184-197 (2017).

4. Charley, P. A. \& Wilusz, J. Standing your ground to exoribonucleases: function of Flavivirus long non-coding RNAs. Virus Res. 212, 70-77 (2016).

5. Charley, P. A., Wilusz, C. J. \& Wilusz, J. Identification of phlebovirus and arenavirus RNA sequences that stall and repress the exoribonuclease XRN1. J. Biol. Chem. 293, 285-295 (2018).

6. Li, Y., Masaki, T., Yamane, D., McGivern, D. R. \& Lemon, S. M. Competing and noncompeting activities of miR-122 and the 5' exonuclease Xrn1 in regulation of hepatitis C virus replication. Proc. Natl Acad. Sci. USA 110, 1881-1886 (2013).

7. Burke, J. M., Kincaid, R. P., Nottingham, R. M., Lambowitz, A. M. \& Sullivan, C. S. DUSP11 activity on triphosphorylated transcripts promotes Argonaute association with noncanonical viral microRNAs and regulates steady-state levels of cellular noncoding RNAs. Genes Dev. 30, 2076-2092 (2016).

8. Amador-Canizares, Y., Bernier, A., Wilson, J. A. \& Sagan, S M. miR-122 does not impact recognition of the HCV genome by innate sensors of RNA but rather protects the $5^{\prime}$ end from the cellular pyrophosphatases, DOM3Z and DUSP11. Nucleic Acids Res. 46, 5139-5158 (2018).

9. Kincaid, R. P., Lam, V. L., Chirayil, R. P., Randall, G. \& Sullivan, C. S. RNA triphosphatase DUSP11 enables exonuclease XRN-mediated restriction of hepatitis C virus. Proc. Natl Acad. Sci. USA 115, 8197-8202 (2018).

10. Luciano, D. J., Vasilyev, N., Richards, J., Serganov, A. \& Belasco, J. G. A novel RNA phosphorylation state enables 5' end-dependent degradation in Escherichia coli. Mol. Cell 67, 44-54 e6 (2017).

11. Deana, A., Celesnik, H. \& Belasco, J. G. The bacterial enzyme RppH triggers messenger RNA degradation by 5' pyrophosphate removal. Nature 451, 355-358 (2008).

12. Schoenberg, D. R. The end defines the means in bacterial mRNA decay. Nat. Chem. Biol. 3, 535-536 (2007).

13. Hsieh, P. K., Richards, J., Liu, Q. \& Belasco, J. G. Specificity of RppHdependent RNA degradation in Bacillus subtilis. Proc. Natl Acad. Sci. USA 110, 8864-8869 (2013).

14. Belasco, J. G. All things must pass: contrasts and commonalities in eukaryotic and bacterial mRNA decay. Nat. Rev. Mol. Cell Biol. 11, 467-478 (2010).

15. Carreras-Puigvert, J. et al. A comprehensive structural, biochemical and biological profiling of the human NUDIX hydrolase family. Nat. Commun. 8, 1541 (2017).
16. Bessman, M. J., Frick, D. N. \& O'Handley, S. F. The MutT proteins or "Nudix" hydrolases, a family of versatile, widely distributed, "housecleaning" enzymes. J. Biol. Chem. 271, 25059-25062 (1996).

17. McLennan, A. G. The Nudix hydrolase superfamily. Cell Mol. Life Sci. 63, 123-143 (2006).

18. Mildvan, A. S. et al. Structures and mechanisms of Nudix hydrolases. Arch. Biochem. Biophys. 433, 129-143 (2005).

19. Stojdl, D. F. et al. VSV strains with defects in their ability to shutdown innate immunity are potent systemic anti-cancer agents. Cancer Cell 4, 263-275 (2003).

20. Ge, H., Chen, X., Yang, W., Niu, L. \& Teng, M. Crystal structure of wild-type and mutant human Ap4A hydrolase. Biochem. Biophys. Res. Commun. 432, 16-21 (2013)

21. Vasilyev, N. \& Serganov, A. Structures of RNA complexes with the Escherichia coli RNA pyrophosphohydrolase RppH unveil the basis for specific 5'-enddependent mRNA decay. J. Biol. Chem. 290, 9487-9499 (2015).

22. Luciano, D. J., Vasilyev, N., Richards, J., Serganov, A. \& Belasco, J. G. Importance of a diphosphorylated intermediate for RppH-dependent RNA degradation. RNA Biol. 15, 703-706 (2018).

23. Pichlmair, A. et al. Activation of MDA5 requires higher-order RNA structures generated during virus infection. J. Virol. 83, 10761-10769 (2009).

24. Schwanhausser, B. et al. Global quantification of mammalian gene expression control. Nature 473, 337-342 (2011).

25. Li, Y., Yamane, D. \& Lemon, S. M. Dissecting the roles of the $5^{\prime}$ exoribonucleases Xrn1 and Xrn2 in restricting hepatitis C virus replication. J. Virol. 89, 4857-4865 (2015)

26. Li, Y., Yamane, D., Masaki, T. \& Lemon, S. M. The yin and yang of hepatitis C: synthesis and decay of hepatitis C virus RNA. Nat. Rev. Microbiol. 13, 544-558 (2015).

27. Wilson, J. A. \& Huys, A. miR-122 promotion of the hepatitis $\mathrm{C}$ virus life cycle: sound in the silence. Wiley Interdiscip. Rev. RNA 4, 665-676 (2013).

28. Choi, J. H. et al. DUSP11-mediated control of 5'-triphosphate RNA regulates RIG-I sensitivity. Genes Dev. 34, 1697-1712 (2020).

29. Goubau, D. et al. Antiviral immunity via RIG-I-mediated recognition of RNA bearing 5'-diphosphates. Nature 514, 372-375 (2014).

30. Marriott, A. S. et al. NUDT2 disruption elevates diadenosine tetraphosphate (Ap4A) and down-regulates immune response and cancer promotion genes. PLoS One 11, e0154674 (2016).

31. Ferron, F., Decroly, E., Selisko, B. \& Canard, B. The viral RNA capping machinery as a target for antiviral drugs. Antivir. Res. 96, 21-31 (2012).

32. Decroly, E., Ferron, F., Lescar, J. \& Canard, B. Conventional and unconventional mechanisms for capping viral mRNA. Nat. Rev. Microbiol. 10, 51-65 (2011).

33. Balvay, L., Soto Rifo, R., Ricci, E. P., Decimo, D. \& Ohlmann, T. Structural and functional diversity of viral IRESes. Biochim. Biophys. Acta 1789, 542-557 (2009).

34. Machlin, E. S., Sarnow, P. \& Sagan, S. M. Masking the $5^{\prime}$ terminal nucleotides of the hepatitis $\mathrm{C}$ virus genome by an unconventional microRNA-target RNA complex. Proc. Natl Acad. Sci. USA 108, 3193-3198 (2011).

35. Romero-Brey, I. \& Bartenschlager, R. Membranous replication factories induced by plus-strand RNA viruses. Viruses 6, 2826-2857 (2014).

36. Pillai, P. S. et al. Mx1 reveals innate pathways to antiviral resistance and lethal influenza disease. Science 352, 463-466 (2016).

37. Zhang, S. Y. et al. TLR3 immunity to infection in mice and humans. Curr. Opin. Immunol. 25, 19-33 (2013).

38. Babiuk, S. et al. TLR9-/- and TLR9+/+ mice display similar immune responses to a DNA vaccine. Immunology 113, 114-120 (2004)

39. Holze, C. et al. Oxeiptosis, a ROS-induced caspase-independent apoptosis-like cell-death pathway. Nat. Immunol. 19, 130-140 (2018).

40. Reuther, P. et al. Generation of a variety of stable Influenza A reporter viruses by genetic engineering of the NS gene segment. Sci. Rep. 5, 11346 (2015).

41. Habjan, M. et al. Sequestration by IFIT1 impairs translation of 2'Ounmethylated capped RNA. PLoS Pathog. 9, e1003663 (2013).

42. Dalton, K. P. \& Rose, J. K. Vesicular stomatitis virus glycoprotein containing the entire green fluorescent protein on its cytoplasmic domain is incorporated efficiently into virus particles. Virology 279, 414-421 (2001).

43. Goldeck, M., Schlee, M., Hartmann, G. \& Hornung, V. Enzymatic synthesis and purification of a defined RIG-I ligand. Methods Mol. Biol. 1169, 15-25 (2014).

44. Arnaud-Barbe, N., Cheynet-Sauvion, V., Oriol, G., Mandrand, B. \& Mallet, F. Transcription of RNA templates by T7 RNA polymerase. Nucleic Acids Res. 26, 3550-3554 (1998).

45. Schaller, T. et al. Analysis of hepatitis $\mathrm{C}$ virus superinfection exclusion by using novel fluorochrome gene-tagged viral genomes. J. Virol. 81, 4591-4603 (2007).

46. Abbas, Y. M., Pichlmair, A., Gorna, M. W., Superti-Furga, G. \& Nagar, B. Structural basis for viral 5'-PPP-RNA recognition by human IFIT proteins. Nature 494, 60-64 (2013).

47. Zhang, C. \& Oldenburg A Simple Statistical Parameter for Use in Evaluation and Validation of High Throughput Screening Assays. J. Biomol. Screen. 4, 67-73 (1999). 
48. Goldeck, M., Tuschl, T., Hartmann, G. \& Ludwig, J. Efficient solid-phase synthesis of pppRNA by using product-specific labeling. Angew. Chem. Int. Ed. Engl. 53, 4694-4698 (2014).

49. Sanjana, N. E., Shalem, O. \& Zhang, F. Improved vectors and genome-wide libraries for CRISPR screening. Nat. Methods 11, 783-784 (2014).

50. Shalem, O. et al. Genome-scale CRISPR-Cas9 knockout screening in human cells. Science 343, 84-87 (2014).

51. Guerra, J. et al. Lysyl-tRNA synthetase produces diadenosine tetraphosphate to curb STING-dependent inflammation. Sci. Adv. https://doi.org/10.1126/ sciadv.aax3333 (2020).

52. Jin, L. et al. MPYS is required for IRF3 activation and type I IFN production in the response of cultured phagocytes to bacterial second messengers c-di-AMP and c-di-GMP1. J. Immunol. 187, 2595-2601 (2011).

53. Katoh, K., Misawa, K., Kuma, K. \& Miyata, T. MAFFT: a novel method for rapid multiple sequence alignment based on fast Fourier transform. Nucleic Acids Res. 30, 3059-3066 (2002).

54. Huson, D. H. et al. Dendroscope: An interactive viewer for large phylogenetic trees. BMC Bioinform. 8, 460 (2007).

55. Robert, X. \& Gouet, P. Deciphering key features in protein structures with the new ENDscript server. Nucleic Acids Res. 42, W320-W324 (2014).

56. Perez-Riverol, Y. et al. The PRIDE database and related tools and resources in 2019: improving support for quantification data. Nucleic Acids Res. 47, D442-D450 (2019)

57. Schrödinger, L. L. The PyMOL Molecular Graphics System, Version 2.3.2, https://pymol.org/2/ (2015).

\section{Acknowledgements}

We thank the core facility of the MPI of biochemistry for support. We further thank Soren Riis Paludan for HSV-1, Gerd Zimmer for VSV-Luc and Fabien Bonneau for advice on the EnzChek assay. Work in the authors' laboratories was supported by an ERC consolidator grant (ERC-CoG ProDAP, 817798), the Federal German Ministry for Education and Research (BMBF; COVINET), the Bavarian State Ministry of Science and Arts (Bavarian Research Network FOR-COVID), and the German Research Foundation (PI 1084/3, PI 1084/4, PI 1084/5 and TRR179/TP10, TRR237/A07) to A.P. Work of P.S. at the Leibniz Institute for Experimental Virology (HPI) was supported by the Free and Hanseatic City of Hamburg and the Federal Ministry of Health. The research of M.B. and D.W. was supported by the German Research Foundation (TRR179).

\section{Author contributions}

B.T.L., K.K., Q.E., L.L.A., A.R., C.D., K.M., A.K., S.M., and P.S. conducted the experiments. B.T.L., K.K., Q.E., L.L.A., and A.R. analyzed the data. J.L., D.W., M.M., and M.B. contributed critical reagents. B.T.L., K.K., Q.E., and A.P. designed the experiments and wrote the paper.

\section{Funding}

Open Access funding enabled and organized by Projekt DEAL.

\section{Competing interests}

The authors declare no competing interests.

\section{Additional information}

Supplementary information The online version contains supplementary material available at https://doi.org/10.1038/s41467-021-27239-y.

Correspondence and requests for materials should be addressed to Andreas Pichlmair

Peer review information Nature Communications thanks Rune Hartmann and the other, anonymous, reviewer(s) for their contribution to the peer review of this work. Peer reviewer reports are available.

Reprints and permission information is available at http://www.nature.com/reprints

Publisher's note Springer Nature remains neutral with regard to jurisdictional claims in published maps and institutional affiliations.

(C) Open Access This article is licensed under a Creative Commons Attribution 4.0 International License, which permits use, sharing, adaptation, distribution and reproduction in any medium or format, as long as you give appropriate credit to the original author(s) and the source, provide a link to the Creative Commons license, and indicate if changes were made. The images or other third party material in this article are included in the article's Creative Commons license, unless indicated otherwise in a credit line to the material. If material is not included in the article's Creative Commons license and your intended use is not permitted by statutory regulation or exceeds the permitted use, you will need to obtain permission directly from the copyright holder. To view a copy of this license, visit http://creativecommons.org/ licenses/by/4.0/

(C) The Author(s) 2021 